\title{
Spin-transfer in an open ferromagnetic layer: from negative damping to effective temperature
}

\author{
J.-E. Wegrowe, M. C. Ciornei, H.-J. Drouhin \\ Laboratoire des Solides Irradiés, Ecole Polytechnique, \\ CNRS-UMR 7642 \& CEA/DSM/DRECAM, 91128 Palaiseau Cedex, France.
}

(Dated: June 5, 2018) 


\begin{abstract}
Spin-transfer is a typical spintronics effect that allows a ferromagnetic layer to be switched by spin-injection. Most of the experimental results about spin transfer (quasi-static hysteresis loops or AC resonance measurements) are described on the basis of the Landau-Lifshitz-Gilbert equation of the magnetization, in which additional current-dependent damping factors are added, and can be positive or negative. The origin of the damping can be investigated further by performing stochastic experiments, like one shot relaxation experiments under spin-injection in the activation regime of the magnetization. In this regime, the Néel-Brown activation law is observed which leads to the introduction of a current-dependent effective temperature. In order to justify the introduction of these counterintuitive parameters (effective temperature and negative damping), a detailed thermokinetic analysis of the different sub-systems involved is performed. We propose a thermokinetic description of the different forms of energy exchanged between the electric and the ferromagnetic sub-systems at a Normal/Ferromagnetic junction.

The derivation of the Fokker-Planck equation in the framework of the thermokinetic theory allows the damping parameters to be defined from the entropy variation and refined with the Onsager reciprocity relations and symmetry properties of the magnetic system. The contribution of the spin-polarized current is introduced as an external source term in the conservation laws of the ferromagnetic layer. Due to the relaxation time separation, this contribution can be reduced to an effective damping. The flux of energy transferred between the ferromagnet and the spin-polarized current can be positive or negative, depending on spin accumulation configuration. The effective temperature is deduced in the activation (stationary) regime, providing that the relaxation time that couples the magnetization to the spin-polarized current is shorter than the relaxation to the lattice.
\end{abstract}

PACS numbers: 72.25.Hg, 75.47.De, 75.40.Gb 
In the context of spintronics, the electrical resistance of magnetic nanostructures are tuned with the magnetization states. Giant magnetoresistance (GMR), or anisotropic magnetoresistance (AMR) allow the magnetization states of nano-layers to be measured with great precision. Such magnetoresistances are easily scalable reading processes and are used for magnetic sensors and random access memorie (MRAM) technology. The possibility of controlling the magnetic configuration of a magnetic nanostructure by injecting spins emerged only in recent studies, opening the way to a readily scalable writing process for MRAMs application. This approach is also extended to thermally assisted switching, in which the heat fluxes are also exploited in order to help the magnetization reversal. In order to control the magnetic configurations and their stabilities (for reading and writing processes), in such magnetic nanopillars, it is necessary to understand on one hand the processes responsible for the magnetization reversal (in the presence of a magnetic field and heat), and on the other hand, the processes governing spin-dependent electronic transport at normal/Ferromagnetic interfaces. Taken separately, both effects are rather well understood today. However, coupling the two processes leads one to consider a large variety of possible mechanisms, called spin-transfer, that involve an ensemble of non-equilibrium subsystems in interaction, with different populations of electrons and different populations of spins. The present work tries to clarify this picture with a phenomenological analysis based on non-equilibrium thermodynamics of open systems.

Magnetization reversal provoked by spin injection has been observed in magnetic nanostructures of various morphologies, from spin-valve multilayers [1, 2, 3, 3, 4, 5, 6, 6] to nanowires 8, 9, 10 or point contacts [11, 12, 13, 14], and different types of magnetic domain walls 15, 16, 17, 18, 19, 20]. In order to describe and interpret these observations, physicists where forced to add one or two current-dependent terms into the well-known dynamical equations that describe a ferromagnetic layer coupled to a heat bath (Fokker-Planck or corresponding Landau-Lifshitz-Gilbert equations). However, the question remains open about the deterministic (e.g. spin-torque) or stochastic (e.g. irreversible) nature of the terms to be added.

It has been observed that for a time window larger than the nanosecond time scale, and in the framework of one-shot measurements (i.e. non-averaged, or irreversible measurements), the magnetization reversal induced by spin-injection is an activated process, with two level fluctuations [21, 22, 23, 24] or simple irreversible jumps [22, 25]. In these experiments, 
governed by stochastic fluctuations and noise, the observed effect is accounted for by a current dependent effective temperature in the Néel-Brown activation law 22]. In contrast, for quasi-static measurements (e.g. magnetoresistance measured as a function of the magnetic field or current with DC systems or lock-in detection system) and for high frequency measurements, oscillations and resonances indicate, in the frequency domain, the manifestation of quasi-ballistic precession effects [14, 24, 26, 27, 28]. In these last experiments, the stochastic nature of the signal is averaged out, and the behavior is described in terms of current dependent negative damping within a generalized Landau-Lifshitz-Gilbert (LLG) equation. This negative damping formulation is motivated by the pioneering works of Berger 29] and Slonczewski 30] about the deterministic spin transfer torque theory. However, the deterministic approach cannot directly account for the magnetic relaxation measurements performed in the activation regime (as discussed in Sec. IV-A below). The hypothesis of the Slonczewski's spin-torque (presented as a current-dependent deterministic term in the microscopic Landau-Lifshitz-Gilbert equation) is not useful as such in the description proposed here, i.e. in the context of open systems.

In order to justify the introduction of the counterintuitive phenomenological parameters (effective temperature and negative damping), a detailed analysis of the different sub-systems is performed on the basis of thermokinetic theory [31, 32, 33, 34, 35, 36, 37, 38, 39, 40, 41, 42, 43, 44]. The first step (first section below) is to identify the relevant sub-systems of interest ( pointing out the difference between the spin-accumulation due to the diffusion of spin-dependent conduction electrons at an interface, and the magnetization of a ferromagnetic layer), the coupling between them, and the role of microscopic degree of freedom that will be reduced to the action of the environment. In section two, spin-injection and spin-dependent transport are described in the framework of the two spin-channel approximation (a conduction channel that carries spin up and a conduction channel that carries spin down, defined by the conductivities). Giant magnetoresistance, spin-accumulation, and corresponding entropy production, or heat transfer, are deduced. Beyond the two spin channel approximation, the analysis is extended to four channels with the introduction of two other electronic populations (typically $s$-like for conduction electrons, and $d$-like for the ferromagnetic order parameter) and the relaxation between them. In the same manner as spin-flip scattering coupled the spin-up and spin-down channels, this relaxation defines a dissipative coupling between the ferromagnet and the spin-dependent electric sub-systems. The third 
section is devoted to the detailed description of the ferromagnetic order parameter coupled to a heat bath (without spin-injection). Both the rotational Fokker-Planck equation and the corresponding LLG equation are derived in the framework of the thermokinetic theory, i.e. with the help of the first two laws of thermodynamics and the Onsager reciprocity relations only. The coupling of the ferromagnetic order parameter to the heat bath is introduced via the chemical potential with a typical Maxwell-Boltzmann diffusion term including the temperature [31, 38]. The Néel-Brown law is deduced in the activation regime.

The last section is devoted to the ferromagnetic Brownian motion activated by spininjection. The contribution of the spin-polarized current is introduced by the $s-d$ like relaxation, as a source term into the conservation laws of the magnetization. Explicitly, it is shown that if $n$ is the density of magnetic moments oriented in a given direction $\theta, \Phi$ of the unit sphere, and $\vec{J}_{M}$ is the corresponding flux of magnetic moments (this flux is not a displacement in the usual space), the conservation of $n$ writes: $\partial n / \partial t=-\operatorname{div} \vec{J}_{M}+\int_{N-F} \dot{\Psi}(z) d z$, where the divergence is defined on the sphere and $\dot{\Psi}$ is the relaxation rate, integrated through the Normal-Ferromagnetic interfaces. This equation defines the irreversible spin-transfer occurring in the ferromagnetic layer, taken as an open system. The relaxation rate is related to the spin-accumulation $\Delta \mu$ through an Onsager transport coefficient $L, \dot{\Psi}=L \Delta \mu$ (where $\Delta \mu$ is proportional to the current). $L$ is linked to the relaxation times through the charge conservation laws (or electric screening properties).

Due to the large relaxation time separation, the contribution of the source term can be reduced to the effect of an environment that is responsible for an effective damping and effective fluctuations (or effective temperature). The energy transferred between ferromagnetic layer and the sub-system defined by the spin-accumulation conduction electrons can be positive or negative, depending on the sign of the spin accumulation at the different interfaces. The effective temperature is deduced in the activation (stationary) regime, because the relaxation time that couples the magnetization to the spin-polarized current short cuts the relaxation to the lattice. 


\section{THERMOKINETIC APPROACH}

\section{A. Interacting sub-systems}

The general scheme of the thermokinetic approach is described in the references 32, 34, 35, 36, 38]. The method consists in defining the state of the system with a set of the relevant extensive variables, say $\left\{s, x_{i}\right\}$, where $x_{i}$ is, e.g. the densities of particles in the sub-system $i$, or equivalently, the density of component $i$ of a multicomponent fluid, and $s$ is the total entropy density. The conservation equations should then be written, and the two laws of thermodynamics applied. The conservation equation for the component $i$ writes:

$$
\frac{\partial n_{i}}{\partial t}=-\operatorname{div}\left(\vec{J}_{i}\right)+\Sigma_{j} \nu_{i j} \dot{\Psi}_{j}
$$

The divergence of the current $\overrightarrow{J_{i}}$ describes the conservative part of the process, and the term $\dot{\Psi}_{j}$ is a source term that describes the relaxation of $\nu_{i j}$ components $i$ into the component $j\left(\nu_{i j} \leq 0\right)$, or inversely $\left(\nu_{i j} \geq 0\right)[45]$. It is proportional to the inverse of the relaxation time $\dot{\Psi}_{j} \propto \tau^{-1}$ (see Appendix A). Physically, the term $\dot{\Psi}_{j}$ describes the relaxation process that changes the internal degree of freedom (e.g. spins, electric charges, internal configuration). In terms of chemical reactions, $\dot{\Psi}_{j}$ is the velocity of the reaction, i.e. the generalized flux thermodynamically conjugated to the chemical affinity $A_{j}$ (defined below). The summation over all sub-systems, or all components of the fluid is that of a conserved variable: $\Sigma_{i} \frac{\partial n_{i}}{\partial t}=-\operatorname{div}\left(\Sigma_{i} \vec{J}_{i}\right)$. The same holds, of course, for the energy $E: \frac{\partial E}{\partial t}=-\operatorname{div}\left(\vec{J}_{E}\right)$, where $J_{E}$ is the flux of energy. In contrast, the entropy production of the total system is not conservative in general, due to the irreversible processes (in other terms, information is lost). The equation for the entropy production of the whole system takes the canonical form $\frac{\partial s}{\partial t}=-\operatorname{div}\left(\vec{J}_{s}\right)+\mathcal{I}$, where $J_{s}$ is the flux of entropy, and $\mathcal{I}$ is the internal entropy production, or irreversibility, which is a consequence of the second law of thermodynamics: $\mathcal{I} \geq 0$ (assuming $T \geq 0$ ). According to the first law of thermodynamics, the energy $E$, is a state function that is also scalar, extensive and conserved, so that

$$
\frac{\partial E\left(s,\left\{x_{i}\right\}\right)}{\partial t}=\frac{\partial E}{\partial s} \frac{\partial s}{\partial t}+\Sigma_{i} \frac{\partial E}{\partial x_{i}} \cdot \frac{\partial x_{i}}{\partial t}
$$

where $\partial E / \partial s=T$ is the temperature, $\partial E / \partial x_{i} \equiv F_{i}$ is the generalized force associated with the flux $\partial x_{i} / \partial t$. In the following we will deal exclusively with the chemical potentials 
$\mu_{i}=\partial n_{i} / \partial E$, unless specified otherwise (i.e. there is no need to introduce other extensive variables). The following Gibbs relation is obtained as a direct consequence of the first law: $T \frac{\partial s}{\partial t}=\frac{\partial E}{\partial t}-\Sigma_{i} \frac{\partial n_{i}}{\partial t} \mu_{i}$. After having inserted the conservation equations, Eq. (10), into Eq. (2), the following form is obtained [46] :

$$
T \frac{\partial s}{\partial t}=-\operatorname{div}\left(\vec{J}_{E}\right)+\Sigma_{i} \mu_{i} \operatorname{div}\left(\overrightarrow{J_{i}}\right)-\Sigma_{i j} \mu_{i} \nu_{i j} \dot{\Psi}_{j}
$$

Using the development $\operatorname{div}\left(\mu_{i} \overrightarrow{J_{i}}\right)=\mu_{i} \operatorname{div}\left(\overrightarrow{J_{i}}\right)+\overrightarrow{J_{i}} \cdot g r a d\left(\mu_{i}\right)$, Eq. (3) can be re-written in the canonical form:

$$
\frac{\partial s}{\partial t}=-\operatorname{div}\left(\overrightarrow{J_{s}}\right)+\mathcal{I}
$$

where:

$$
\left\{\begin{array}{l}
\vec{J}_{s}=\frac{1}{T} \vec{J}_{E}-\Sigma_{i} \frac{\mu_{i}}{T} \vec{J}_{i} \\
\mathcal{I}=\vec{J}_{E} \cdot \operatorname{grad}\left(\frac{1}{T}\right)-\Sigma_{i} \overrightarrow{J_{i}} \cdot \operatorname{grad}\left(\frac{\mu_{i}}{T}\right)-\frac{1}{T} \Sigma_{i j} \mu_{i} \nu_{i j} \dot{\Psi}_{j}
\end{array}\right.
$$

where the last term on the right hand side defines the dissipative coupling between the sub-systems. As will be shown in the last section, this term is responsible for the irreversible spin-transfer effect described in this work. What is unusual in dealing with the second law, is to manipulate an inequality instead of an equality, and consequently to deal with sufficient conditions instead of equivalences. Here, the condition $\mathcal{I} \geq 0$ leads to a positive matrix $\left\{\mathcal{L}_{i j}\right\}_{i j}$ of Onsager-Casimir transport coefficients that are state functions of the variables $\left\{s, x_{i}\right\}$, in order to build a positive quadratic form. The condition is fulfilled if the flux $J_{i}$ and the relaxation velocity $\dot{\Psi}_{i}$ have the form

$$
\left\{\begin{array}{l}
\vec{J}_{i}=-\Sigma_{j} \mathcal{L}_{i j} \operatorname{grad}\left(\mu_{j}\right) \\
\dot{\Psi}_{i}=\Sigma_{j} L_{i j} A_{j}
\end{array}\right.
$$

where

$$
A_{j} \equiv-\Sigma_{k} \nu_{i k} \mu_{k}
$$

is the chemical affinity of the corresponding reaction $j$ (and we have $A_{j}=-\partial E / \partial \Psi_{j}$ ) [47]. Furthermore, due to the time reversal symmetry of the microscopic equations, the transport 
coefficients follow the Onsager-Casimir reciprocity relations 48]. The cross-coefficients that couple the flux $\vec{J}$ to the relaxation process $\dot{\Psi}$ are assumed to be zero, because, according to the Curie principle, only processes of identical tensorial nature are coupled. Inserting Eq. (6) into the continuity equation Eq. (11), we obtain an equation of the time variation of the density $\partial n_{i} / \partial t$ in terms of derivatives of the chemical potentials $\mu_{j}$ :

$$
\frac{\partial n_{i}}{\partial t}=\Sigma_{j} \mathcal{L}_{i j} \nabla^{2} \mu_{j}+\Sigma_{j k} \nu_{i j} L_{i k} A_{k}
$$

It is then sufficient to know the form of the chemical potential as a function of the density (for pur fluids : $\left.\mu\left(n_{i}\right)=\mu_{0}+k T \ln \left(n_{i} / N\right)\right)$ in order to derive the corresponding differential equation, or Fokker-Planck equation, with diffusion and relaxation terms (see sections II, III and VI below).

What we gain in performing this analysis is to identify clearly the conservative and dissipative flux (through the internal entropy production), and to be able to define a dissipative process that couples the sub-systems beyond the usual deterministic coupling (electric field, magnetic field, pressure, etc...). This dissipative coupling appears with an additional transport coefficient $L$, defined univocally via the transport equations. In the case studied below, the matrix $\mathcal{L}$ is composed by theßconductivities $\sigma_{i}$ associated to each channel (i.e. associated to a given electronic population), the thermal conductivity, or the corresponding Seebeck (thermoelectric power) and Peltier coefficients [43, 49, 50] and the ferromagnetic transport coefficients: gyromagnetic ratio $\Gamma$ and the Gilbert damping coefficient $\eta$. Beyond, the flux of entropy or heat allows the spin transfer to be understood in an open system in terms of relaxation with a supplementary Onsager coefficient $L$. As shown in the last section, this term is responsible for an effective temperature $T_{\text {eff }}$ and effective (negative) damping $\alpha_{e f f}$.

\section{B. The model}

The model is based on the hypothesis that the ferromagnetic order parameter $\vec{M}$ is well differentiated from the sub-system composed by spin-polarized conduction electrons, although both systems exchange charges, spins, and heat through a relaxation mechanism that will be described in terms of internal variables [31, 35, 38]. As shown above, the relaxation of an internal variable (or internal degree of freedom) defines a transport coefficient $L_{s d}$ related to the corresponding relaxation time $\tau_{s d}\left(L_{s d} \propto \tau_{s d}^{-1}\right.$, see appendix $\mathrm{A}$ for the 
relation to the relaxation time).

We hence start with the two sub-systems: the ferromagnet described by the magnetization $\vec{M}$ and the two conducting spin-channel system of the conduction electrons. Both subsystems are dynamically coupled through the relaxation time $\tau_{s d}$. This relaxation is qualified as interband relaxation, to be opposed to the intraband spin-flip relaxation $\tau_{s f}$ introduced in the usual two spin-channel approximation. The conducting channels are usually described by the density $n_{\uparrow}$ of conduction electrons with spin up and the density $n_{\downarrow}$ of conduction electrons with spin down. The intraband coupling (accounted for by $L_{s f}$ or $\tau_{s f}$ ) is responsible for the spin-accumulation mechanism at stationary regime. For convenience, we redefine the two channels with the density of spin-polarized electrons $\Delta n=n_{\uparrow}-n_{\downarrow}$ ("spin conduction channel") and the total density of electrons $n_{0}=n_{\uparrow}+n_{\downarrow}$.

Furthermore, the conduction channels are contacted to a power supply (current generator here). Strictly speaking, the magnetic system is also contacted to the power supply, e.g. through the electron of $d$ character [51]. The conduction electrons are thermalized eachother through a well-known mechanism of elastic scattering $\tau_{e}$ (that defines the conduction electron reservoir), at the femto-second time scales (or below), and are also contacted to the lattice through the Fermi-Dirac distribution, and inelastic scattering $\tau_{p h}$. On the other hand, the ferromagnetic order parameter is contacted to the lattice with a well-known relaxation time $\tau_{0}$ that is measured in ferromagnetic resonance (FMR) experiments, and is typically of the order of the nanosecond (or few hundreds of picoseconds). This description leads to the model depicted in Fig 1(b).

The basic idea developed below lays on the fact that the typical time scales of the dynamics of the two sub-systems are largely separated. There is a slow variable, the magnetization, and fast variables, the degree of freedom related to the spin of the conduction electrons. It is then possible to reduce the action of the fast variable to the role of an environment with regard to the magnetization, like for spin-bath relaxation. The effect of the coupling to the spin-dependent electronic sub-system will then be reduced to specific damping and fluctuation terms added to the usual stochastic equations for the magnetization. This will be our line of reasoning followed in the last section, after describing the two sub-systems. 


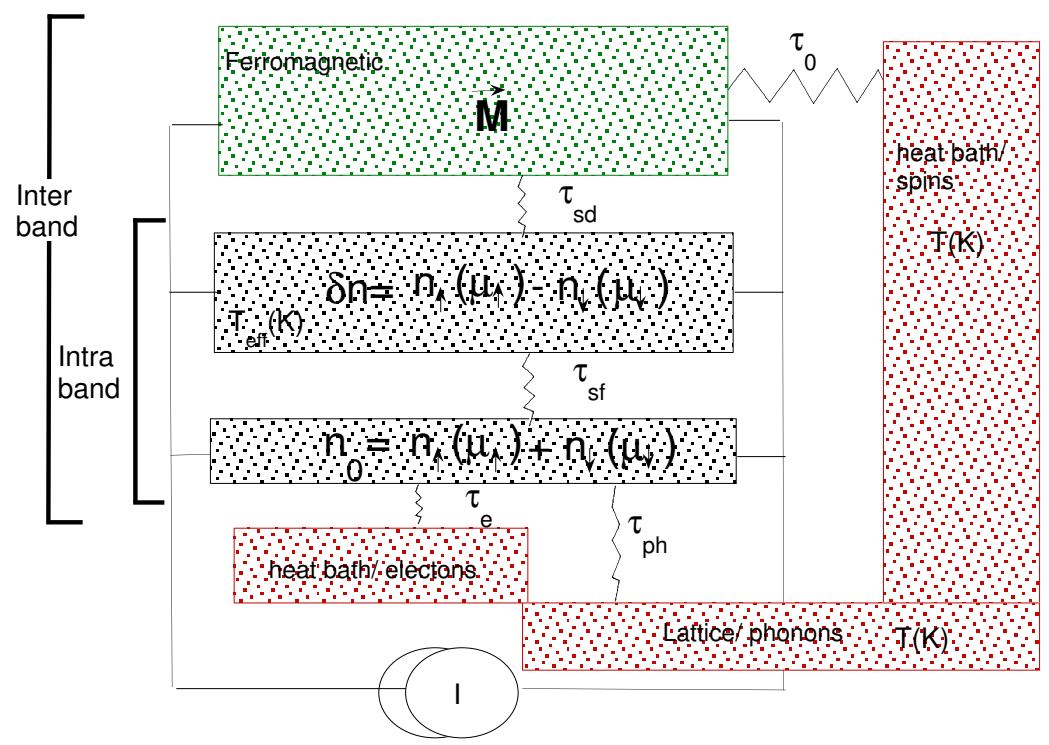

FIG. 1: Thermokinetic picture of irreversible spin-transfer. Ferromagnetic system (with magnetization $\mathrm{M}$ ), and electric system with spin accumulation density $\Delta n$ and electronic density at the Fermi level $n_{0}$. The chemical potential $\mu$ is defined for each spin channel. The three subsystems are coupled together through the relaxation times $\tau_{s d}$ (interband $s-d$ like relaxation) and $\tau_{s f}$.(intraband spin-flip relaxation). The sub-systems are also coupled to the current generator $I$, and to the heat reservoirs, through the corresponding well known relaxation times $\tau_{0}$ (Néel-Brown waiting time), $\tau_{e}$ and $\tau_{p h}$ : elastic and inelastic electronic relaxation times.

\section{SPIN-DEPENDENT TRANSPORT}

In order to explain the high resistance and the high thermoelectric power observed in transition metals, Mott introduced the concept of spin-polarized current and suggested that s-d interband scattering plays an essential role in the conduction properties [52]. This approach in terms of two conduction bands [51], explained the existence of a spin-polarized current in the $3 \mathrm{~d}$ ferromagnetic materials [53], and was used for the description of anisotropic magnetoresistance (AMR) [54, 55], the description of spin-polarizer [56], and thermoelectric power [57]. With the discovery of giant magnetoresistance (GMR) [58] and related effects 59] (like domain wall scattering [60, 61, 62, 63] discussed below ), the development of spintronics focused the discussion on spin-flip scattering occurring between spin-polarized conducting channels [64, 65, 66, 67, 68, 69]. The two-channel model, which describes the 
conduction electrons with majority and minority spins, is applied with great efficiency to GMR and spin injection effects [42, 70, 71, 72, 73, 74, 75], including metal/semiconductor 76] and metal/superconductor interfaces [77]. In this context, it is sufficient to describe the diffusion process in terms of spin-flip scattering without the need to invoke interband s-d scattering.

It is convenient to generalize the two spin channel approach to any relevant transport channels, i.e. to any distinguishable electron populations $\alpha$ and $\gamma$ (defined by an internal degree of freedom). The local out-of-equilibrium state near the junction is then described by a non-vanishing chemical-potential difference between these two populations: $\Delta \mu_{\alpha \gamma}=$ $\mu_{\alpha}-\mu_{\gamma} \neq 0$. In other words, assuming that the presence of a junction induces a deviation from the local equilibrium, the $\alpha$ and $\gamma$ populations can be defined by the $\alpha \rightarrow \gamma$ relaxation mechanism itself, that allows the local equilibrium to be recovered in the bulk material $\left(\lim _{z \rightarrow \pm \infty} \Delta \mu(z)=0\right)$ [42]. Such considerations have been presented in some important spintronics studies on the basis of microscopic calculations [52, 54, 55, 64, 66, 74, 75, 78,

79, 80]. The thermokinetic approach [81] allows us to deal with interband relaxation on an equal footing with spin-flip relaxation, with the help of the transport coefficients only. For this purpose, the two spin-channel model is generalized, with the introduction of the corresponding transport coefficients: the conductivities $\sigma_{\alpha}$ and $\sigma_{\gamma}$ of each channel define the total conductivity $\sigma_{t}=\sigma_{\alpha}+\sigma_{\gamma}$ and the conductivity asymmetry $\beta=\left(\sigma_{\alpha}-\sigma_{\gamma}\right) / \sigma_{t}$; the relaxation between both channels is described by the parameter $L$ (or equivalently, the relevant relaxation times $\left.\tau_{\gamma \leftrightarrow \alpha}\right)$.

\section{A. The generalized two channel model}

In the framework of the two conducting-channel model, which includes relaxation from one channel to the other, it easy to follow step by step the method described in the first section. The conservation laws write (assuming a 1D space variable $z$ ):

$$
\left\{\begin{array}{l}
\frac{\partial n_{\alpha}}{\partial t}=-\frac{\partial J_{\alpha}}{\partial z}-\dot{\Psi}_{\alpha \gamma} \\
\frac{\partial n_{\gamma}}{\partial t}=-\frac{\partial J_{\gamma}}{\partial z}+\dot{\Psi}_{\alpha \gamma}
\end{array}\right.
$$

where $n_{\alpha}$ and $n_{\gamma}$ are the densities of particles in the channels $\{\alpha, \gamma\}$. 


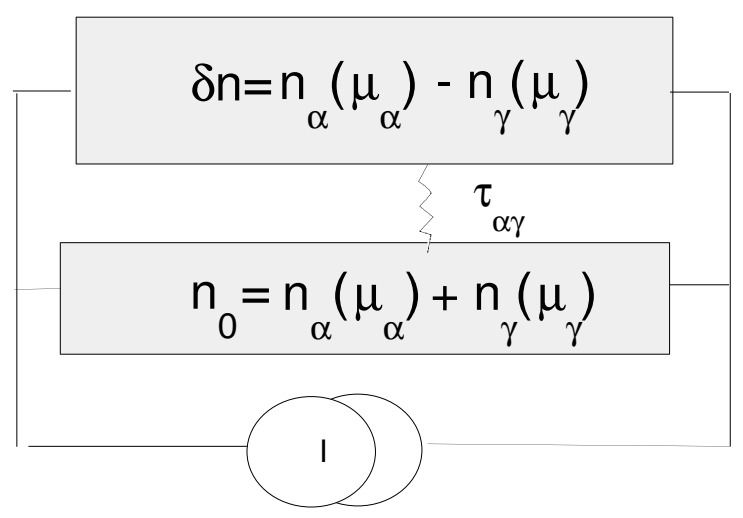

FIG. 2: Two channel model, including relaxation that couples the two electronic populations.

The entropy variation writes:

$$
T \mathcal{I}=-J_{\alpha} \frac{\partial \mu_{\alpha}}{\partial z}-J_{\gamma} \frac{\partial \mu_{\gamma}}{\partial z}-\dot{\Psi}_{\alpha \gamma}\left(\mu_{\alpha}-\mu_{\gamma}\right)
$$

the application of the second law of thermodynamics leads to introduce the Onsager coefficients $\sigma_{\alpha} \geq 0, \sigma_{\gamma} \geq 0$, and $L \geq 0$ [42, 81], such that:

$$
\left\{\begin{array}{c}
J_{\alpha}=-\frac{\sigma_{\alpha}}{e} \frac{\partial \mu_{\alpha}}{\partial z} \\
J_{\gamma}=-\frac{\sigma_{\gamma}}{e} \frac{\partial \mu_{\gamma}}{\partial z} \\
\dot{\Psi}_{\alpha \gamma}=L\left(\mu_{\alpha}-\mu_{\gamma}\right)
\end{array}\right.
$$

where $\dot{\Psi}_{\alpha \gamma}$ describes the relaxation from the channel $\alpha$ to the other channel $\gamma$ in terms of velocity of the reaction $\alpha \rightarrow \gamma$. It is not necessary, in what follows, to distinguish between the electric part and the pure chemical part of the electro-chemical potentials (see [82] ). The effects of the electric charge distribution are described in Appendix A, with the introduction of the screening length $l$ and the relation to the relaxation times. As shown in Appendix A, the Onsager coefficient $L$ is inversely proportional to the electronic relaxation times $\tau_{\alpha \leftrightarrow \gamma}$ :

$$
L \propto\left(\frac{g}{\tau_{\alpha \rightarrow \gamma}}+\frac{f}{\tau_{\gamma \rightarrow \alpha}}\right)
$$

where $f$ and $g$ are two functions close to unity, and related to the electric charge distributions (see Appendix A). Note that due to our definition of $\mu_{\alpha}$ and $\mu_{\gamma}$, there is no direct coupling between the two channels : there is no transport coefficients that couples the two first equations in Eq. (11). This is a consequence of the definition of the electronic populations, through the relaxation process itself (the populations are stable if $\dot{\Psi}=0$ ). Indeed, 


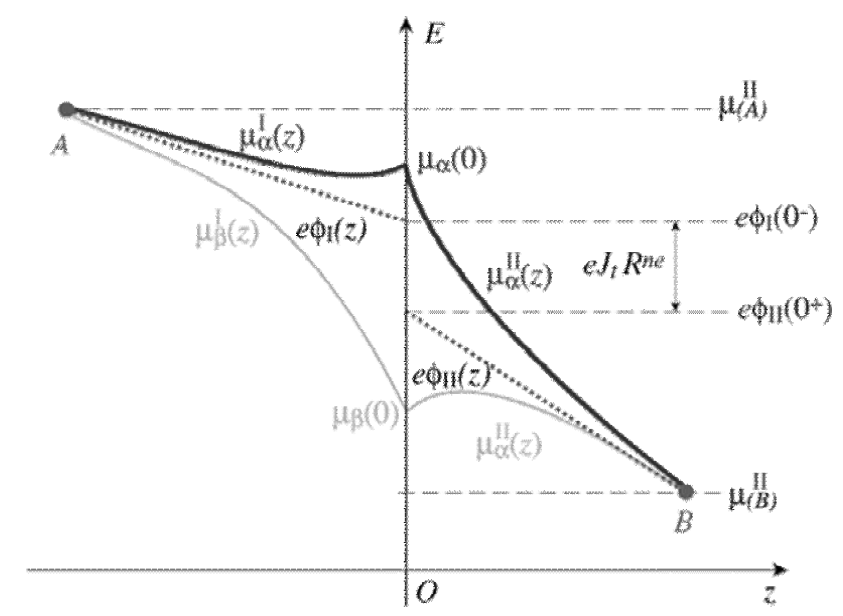

FIG. 3: Junction between to layers $I$ and $I I$. Chemical potential profile over the interval $[A, B]$ in the $\alpha$ and $\gamma$ channels. The A and B points verify $\mu_{\alpha}(A)=\mu_{\gamma}(A)$ and $\mu_{\alpha}(B)=\mu_{\gamma}(B)$. The two straight lines represent the $\Phi$ variation in each region $\left(\Phi_{I}, \Phi_{I I}\right)$. It can be directly seen that the out-of-equilibrium resistance $R^{\text {ne }}$ is determined by the $\Phi$ discontinuity at the interface.

the out-of-equilibrium configuration at the interface is quantified by the chemical affinity $\Delta \mu=\mu_{\alpha}-\mu_{\gamma}$, i.e. the chemical potential difference of the reaction.

The total current $J_{t}$ is constant:

$$
J_{t}=J_{\alpha}+J_{\gamma}=-\frac{1}{e} \frac{\partial}{\partial z}\left(\sigma_{\alpha} \mu_{\alpha}+\sigma_{\gamma} \mu_{\gamma}\right)
$$

However, it is not possible to measure separately the different conduction channels, since any realistic electric contact short-cuts the two channels. What is measured is necessarily the usual Ohm's law, $J_{t}=-\sigma_{t} \frac{\partial \Phi}{\partial z}$, that imposes the reference electric potential $\Phi$ to be introduced, together with the total conductivity $\sigma_{t}=\sigma_{\alpha}+\sigma_{\gamma}[83]$. The potential $\Phi$ is hence:

$$
e \Phi=\frac{1}{\sigma_{t}}\left(\sigma_{\alpha} \mu_{\alpha}+\sigma_{\gamma} \mu_{\gamma}\right)
$$

Let us assume that the two channels collapse to a unique conduction channel for a specific configuration, the reference, which is a local equilibrium situation: $\Delta \mu_{e q}=0$. The out-ofequilibrium contribution to the resistance, $R^{n e}$, is calculated through the relation:

$$
J_{t} e R^{n e}=\int_{A}^{B} \frac{\partial}{\partial z}\left(\mu_{\alpha}-e \Phi(z)\right) d z=\int_{A}^{B} \frac{\partial}{\partial z}\left(\mu_{\gamma}-e \Phi(z)\right) d z
$$


so that

$$
R^{n e}=-\frac{1}{J_{t} e} \int_{A}^{B} \frac{\sigma_{\alpha}-\sigma_{\gamma}}{2 \sigma_{t}} \frac{\partial \Delta \mu}{\partial z} d z
$$

where the measurement points $A$ and $B$ are located far enough from the interface (inside the bulk) so that $\Delta \mu(A)=\Delta \mu(B)=0$ (see Fig. 3). The integral in Eqs. (15) is performed over the regular part of the function only ( $\Phi$ and $\sigma_{i}$ are discontinuous) 84]. Eq. [16] allows the out-of-equilibrium resistance at a simple junction between two layers (composed by the layers $I$ and $I I)$ to be easily calculated. If the junction is set at $z=0$ and the conductivities are respectively $\sigma_{i}^{I}$ and $\sigma_{i}^{I I}(i=\{\alpha, \gamma\})$, we have:

$$
J_{T} e R^{n e}=\int_{A}^{0} \frac{\sigma_{\alpha}^{I}-\sigma_{\gamma}^{I}}{2 \sigma_{t}} \frac{\partial \Delta \mu^{I}}{\partial z} d z+\int_{0}^{B} \frac{\sigma_{\alpha}^{I I}-\sigma_{\gamma}^{I I}}{2 \sigma_{t}} \frac{\partial \Delta \mu^{I I}}{\partial z} d z
$$

The equilibrium is recovered in the bulk, so that:

$$
R^{n e}=\left(\frac{\sigma_{\alpha}^{I}-\sigma_{\gamma}^{I}}{\sigma_{t}^{I}}-\frac{\sigma_{\alpha}^{I I}-\sigma_{\gamma}^{I I}}{\sigma_{t}^{I I}}\right) \frac{\Delta \mu(0)}{2 J_{t} e}
$$

The chemical potential difference $\Delta \mu(z)$, which accounts for the pumping force opposed to the relaxation $\alpha \rightarrow \gamma$, is obtained by solving the diffusion equation deduced from Eqs. (111) and (91), and assuming a stationary regime for each channels, $\frac{\partial n_{\alpha}}{\partial t}=\frac{\partial n_{\gamma}}{\partial t}=0$ [42, 70, 71, 72, 73]:

$$
\frac{\partial^{2} \Delta \mu(z)}{\partial z^{2}}=\frac{\Delta \mu(z)}{l_{d i f f}^{2}}
$$

where

$$
l_{\text {diff }}^{-2}=e L\left(\sigma_{\alpha}^{-1}+\sigma_{\gamma}^{-1}\right)
$$

is the diffusion length related to the $\alpha \rightarrow \gamma$ relaxation.

At the interface $(z=0)$, the continuity of the currents for each channel writes $J_{\alpha}^{I}(0)=$ $J_{\alpha}^{I I}(0)$, were

$$
J_{\alpha}(0)=-\frac{\sigma_{\alpha} \sigma_{\gamma}}{e \sigma_{t}} \frac{\partial \Delta \mu}{\partial z}+\frac{\sigma_{\alpha}}{\sigma_{t}} J_{t}
$$

which leads to the general relation:

$$
\Delta \mu(0)=\left(\frac{\sigma_{\alpha}^{I}}{\sigma_{t}^{I}}-\frac{\sigma_{\alpha}^{I I}}{\sigma_{t}^{I I}}\right)\left(\frac{\sigma_{\alpha}^{I} \sigma_{\gamma}^{I}}{\sigma_{t}^{I} l_{\text {diff }}^{I}}+\frac{\sigma_{\alpha}^{I I} \sigma_{\gamma}^{I I}}{\sigma_{t}^{I I} l_{\text {diff }}^{I I}}\right)^{-1} e J_{t}
$$


Inserting Eq. (22) into Eq. (18), we obtain the general expression for the out-ofequilibrium resistance (per unit area) produced by the $\alpha \rightarrow \gamma$ relaxation mechanism at a junction:

$$
R^{n e}=\left(\frac{\sigma_{\alpha}^{I}-\sigma_{\gamma}^{I}}{2 \sigma_{t}^{I}}-\frac{\sigma_{\alpha}^{I I}-\sigma_{\gamma}^{I I}}{2 \sigma_{t}^{I I}}\right)\left(\frac{\sigma_{\alpha}^{I}}{\sigma_{t}^{I}}-\frac{\sigma_{\alpha}^{I I}}{\sigma_{t}^{I I}}\right)\left(\sqrt{\frac{\sigma_{\alpha}^{I} \sigma_{\gamma}^{I} e L^{I}}{\sigma_{t}^{I}}}+\sqrt{\frac{\sigma_{\alpha}^{I I} \sigma_{\gamma}^{I I} e L^{I I}}{\sigma_{t}^{I I}}}\right)^{-1}
$$

where we have used the relation :

$$
l_{\text {diff }}^{-1}=2 \sqrt{\frac{e L}{\sigma_{t}\left(1-\beta^{2}\right)}}
$$

It is convenient to describe the conductivity asymmetry by a parameter $\beta$ such that $\sigma_{\alpha}=\sigma_{t}(1+\beta) / 2$ and $\sigma_{\gamma}=\sigma_{t}(1-\beta) / 2$. The out-of-equilibrium contribution to the resistance then takes the following form:

$$
R^{n e}=\frac{1}{2} \frac{\left(\beta_{I}-\beta_{I I}\right)^{2}}{\sqrt{e L^{I} \sigma_{t}^{I}\left(1-\beta_{I}^{2}\right)}+\sqrt{e L^{I I} \sigma_{t}^{I I}\left(1-\beta_{I I}^{2}\right)}}
$$

In the case of the subsystem described by two spin-channel, the relaxation $\dot{\Psi}_{\uparrow \downarrow}$ leads to a spin-accumulation effect $\Delta \mu_{\uparrow \downarrow}$ at the interface of a two identical ferromagnet with antiparallel configuration. The corresponding resistance contribution is:

$$
R_{s a}^{\uparrow \downarrow}=\frac{\beta_{s}^{2}}{\sigma_{t}\left(1-\beta_{s}^{2}\right)} l_{s f}=\frac{\beta_{s}^{2}}{\sqrt{e L \sigma_{t}\left(1-\beta_{s}^{2}\right)}}
$$

This expression is the well-known giant magnetoresistance contribution [42, 70, 71, 72, 73, 85, 86].

\section{B. The four channel approximation}

In the previous subsections, two different electronic relaxation mechanisms have been invoked separately in order to describe giant magnetoresistance or anisotropic magnetoresistance. It is clear however that the two relaxations would take place in parallel, leading to a more complex redistribution of spins within the different channels. In the present subsection, we consider a system in which the two mechanisms coexist, leading to a four channel model [4]. 
The generic band structure (energy as a function of wave vector $\vec{k}$ for a given direction) of a $3 \mathrm{~d}$ ferromagnet is schematized in Fig. 4. The band $\mathrm{s}$ is parabolic and the exchange splitting is very small. In contrast, the d bands are strongly shifted between up and down spin carriers. The hybridized zone is schematized by the dotted lines at the intersection.

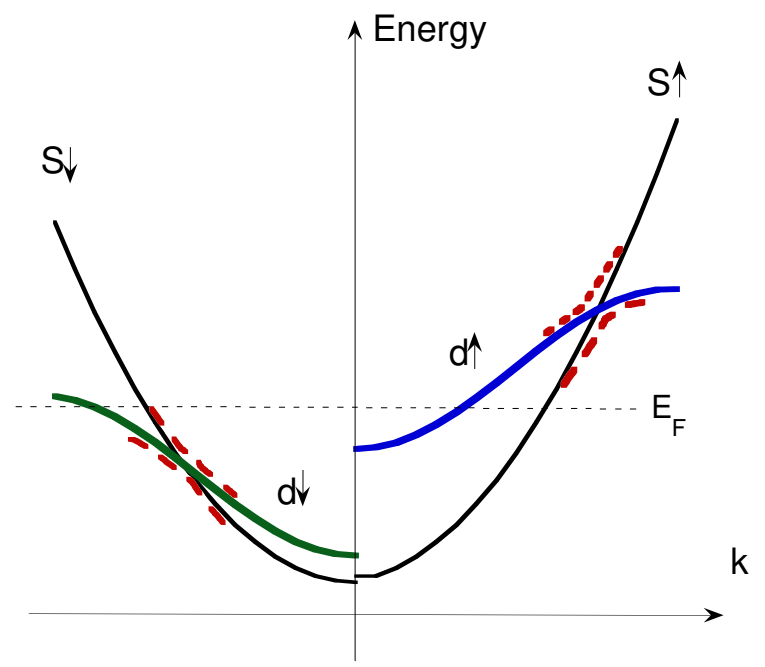

FIG. 4: Generic band structure for a 3d ferromagnet with s and d bands schematized for an arbitrary direction of the wave vector $\mathrm{k}$. The shift between the two $\mathrm{d}$ bands for the two spin carriers up and down is exemplified. The hybridized zone is schematized with dotted lines at the junction between s and d bands. At the Fermi level four different electronic populations can be identified.

The system is composed by the reservoirs of the injected $s$ electrons and the ferromagnetic layer composed by the $d$ electrons. At the interface, current injection leads to a redistribution of the different electronic populations that are governed by spin polarization and charge conservation laws. Let us assume that the current injected is spin polarized in the down polarization $(\downarrow)$. The conservation laws should be written by taking into account the reaction mechanisms between the different populations. At short time scales (electronic scattering) the relaxation channels are assumed to be the following four

(I) $e_{s \downarrow} \rightarrow e_{d \downarrow}$ (spin-conserved $s$ - $d$ scattering) 
(II) $e_{s \downarrow} \rightarrow e_{s \uparrow}$ (spin-flip scattering for the $s$ population)

(III) $e_{s \downarrow} \rightarrow e_{d \uparrow}$ (spin-flip $s$ - $d$ scattering)

(IV) $e_{d \downarrow} \rightarrow e_{d \uparrow}$ (spin-flip scattering for the $d$ population)

Process (I) is assumed to be the main mechanism responsible for anisotropic magnetoresistance (AMR). Process (II) leads to the well-known spin-accumulation effect and was also described in detail in the first subsections. According to the fact that the majority-spin $d$ band is full and lies at a sizable energy below the Fermi level, the current $J_{d^{\wedge}}$ is negligible and the channel $d \uparrow$ is frozen. Processes (III) and (IV) are hence negligible [56]. Consequently, we are dealing with a three-channel model $\{s \uparrow, s \downarrow, d \downarrow\}$.

The total current $J_{t}$ is composed by the three currents for each channel : $J_{t}=J_{s \uparrow}+$ $J_{s \downarrow}+J_{d \downarrow}$. In order to write the conservation laws, the relaxation rate $\dot{\Psi}_{s d}$, is introduced to account for $s-d$ spin-conserved scattering, and the relaxation rate $\dot{\Psi}_{s}$, is introduced in order to account for spin-flip scattering. Assuming that all channels are in a steady state (this condition will relax in the last section, where the magnetic system is coupled to the channels $d \downarrow)$ :

$$
\left\{\begin{aligned}
\frac{\partial n_{t}}{\partial t} & =-\frac{\partial J_{t}}{\partial z}=0 \\
\frac{\partial n_{s \uparrow}}{\partial t} & =-\frac{\partial J_{s \uparrow}}{\partial z}-\dot{\Psi}_{s}=0 \\
\frac{\partial n_{s \downarrow}}{\partial t} & =-\frac{\partial J_{s \downarrow}}{\partial z}-\dot{\Psi}_{s d}+\dot{\Psi}_{s}=0 \\
\frac{\partial n_{d \downarrow}}{\partial t} & =-\frac{\partial J_{d \downarrow}}{\partial z}+\dot{\Psi}_{s d}=0
\end{aligned}\right.
$$

where $n_{t}, n_{s \uparrow}, n_{s \downarrow}, n_{d \downarrow}$ are respectively the total densities of particles and the density of particles in the in the $s \uparrow, s \downarrow, d \downarrow$ channels. The system is described by the number of electrons present in each channel at a given time, that defines the four currents, plus the entropy of the system. The conjugate (intensive) variables are the chemical potentials $\left\{\mu_{s \uparrow}, \mu_{s \downarrow}, \mu_{d \uparrow}, \mu_{d \downarrow}\right\}$. As described in Appendix B, the application of the first and second laws 
of thermodynamics allows us to deduce the Onsager relations of the system :

$$
\left\{\begin{array}{l}
J_{s \downarrow}=-\frac{\sigma_{s \downarrow}}{e} \frac{\partial \mu_{s \downarrow}}{\partial z} \\
J_{s \uparrow}=-\frac{\sigma_{s \uparrow}}{e} \frac{\partial \mu_{s \uparrow}}{\partial z} \\
J_{d \downarrow}=-\frac{\sigma_{d \downarrow}}{e} \frac{\partial \mu_{d \downarrow}}{\partial z} \\
\dot{\Psi}_{s d}=L_{s d}\left(\mu_{s \downarrow}-\mu_{d \downarrow}\right) \\
\dot{\Psi}_{s}=L_{s}\left(\mu_{s \uparrow}-\mu_{s \downarrow}\right)
\end{array}\right.
$$

where the conductivity of each channel $\left\{\sigma_{s \uparrow}, \sigma_{s \downarrow}, \sigma_{d \uparrow}, \sigma_{d \downarrow}\right\}$ has been introduced. The first four equations are nothing but Ohm's law applied to each channel, and the two last equations introduce new Onsager transport coefficients (see Appendix B), $L_{s d \downarrow}$ and $L_{s}$, that respectively describe the $s-d$ relaxation (I) for minority spins under the action of the chemical potential difference $\Delta \mu_{\downarrow}=\mu_{s \downarrow} / 2-\mu_{d \downarrow}$ and the spin-flip relaxation (II) under spin pumping $\Delta \mu_{s}=\mu_{s \uparrow}-\mu_{s \downarrow} / 2$. According to Appendix A, the Onsager coefficients are proportional to the corresponding relaxation times.

For convenience, we define the usual charge current $J_{0 s}=J_{s \uparrow}+J_{s \downarrow}$, the minority-spin current $J_{0 \downarrow}=J_{s \downarrow}+J_{d \downarrow}$, and the two polarized currents $\delta J_{\downarrow}=J_{s \downarrow}-J_{d \downarrow}$ and $\delta J_{s}=J_{s \uparrow}-J_{s \downarrow}$. We introduce the $\sigma_{s}$ and $\sigma_{\uparrow}$ conductivities $\left\{\sigma_{s}=\sigma_{s \uparrow}+\sigma_{s \downarrow}\right.$ and $\left.\sigma_{\downarrow}=\sigma_{s \downarrow}+\sigma_{d \downarrow}\right\}$. The conductivity imbalance $\beta_{\downarrow}$ and $\beta_{s}$ between respectively the $s \downarrow$ and $d \downarrow$ channels and the $s \uparrow$ and $s \downarrow$ channels are:

$$
\left\{\begin{array}{l}
\beta_{\downarrow}=\frac{\sigma_{s \downarrow}-\sigma_{d \downarrow}}{\sigma_{\downarrow}} \\
\beta_{s}=\frac{\sigma_{s \uparrow}-\sigma_{s \downarrow}}{\sigma_{s}}
\end{array}\right.
$$

Eqs. (27) becomes :

$$
\left\{\begin{aligned}
\frac{\partial J_{t}}{\partial z} & =\frac{\partial J_{d \downarrow}}{\partial z}+\frac{\partial J_{s}}{\partial z}=0 \\
\frac{\partial J_{0 \downarrow}}{\partial z} & =\dot{\psi}_{s} \\
\frac{\partial \delta J_{\downarrow}}{\partial z} & =-2 \dot{\psi}_{s d}-\dot{\psi}_{s} \\
\frac{\partial J_{0 s}}{\partial z} & =-\dot{\psi}_{s d} \\
\frac{\partial \delta J_{s}}{\partial z} & =\dot{\psi}_{s d}-2 \dot{\psi}_{s}
\end{aligned}\right.
$$


and, defining the quasi-chemical potentials $\mu_{s}=\mu_{s \uparrow}+\mu_{s \downarrow} / 2$ and $\mu_{\downarrow}=\mu_{s \downarrow} / 2+\mu_{d \downarrow}$, Eqs. (28) becomes :

$$
\left\{\begin{array}{l}
J_{0 \downarrow}=-\frac{\sigma_{\downarrow}}{2 e}\left(\frac{\partial \mu_{\downarrow}}{\partial z}+\beta_{\downarrow} \frac{\partial \Delta \mu_{\downarrow}}{\partial z}\right) \\
\delta J_{\downarrow}=-\frac{\sigma_{\downarrow}}{2 e}\left(\beta_{\downarrow} \frac{\partial \mu_{\downarrow}}{\partial z}+\frac{\partial \Delta \mu_{\downarrow}}{\partial z}\right) \\
J_{0 s}=-\frac{\sigma_{s}}{2 e}\left(\frac{\partial \mu_{s}}{\partial z}+\beta_{s} \frac{\partial \Delta \mu_{s}}{\partial z}\right) \\
\delta J_{s}=-\frac{\sigma_{s}}{2 e}\left(\beta_{s} \frac{\partial \mu_{s}}{\partial z}+\frac{\partial \Delta \mu_{s}}{\partial z}\right) \\
\dot{\Psi}_{s d}=L_{s d} \Delta \mu_{\downarrow} \\
\dot{\Psi}_{s}=L_{s} \Delta \mu_{s}
\end{array}\right.
$$

The equations of conservation [Eqs. (30)] and the above Onsager equations lead to the two coupled diffusion equations :

$$
\left\{\begin{array}{l}
\frac{\partial^{2} \Delta \mu_{\downarrow}}{\partial z^{2}}=\frac{1}{l_{s d}^{2}} \Delta \mu_{\downarrow}-\frac{1}{\lambda_{s}^{2}} \Delta \mu_{s} \\
\frac{\partial^{2} \Delta \mu_{s}}{\partial z^{2}}=\frac{1}{\lambda_{s d}^{2}} \Delta \mu_{\downarrow}-\frac{1}{l_{s f}^{2}} \Delta \mu_{s}
\end{array}\right.
$$

where

$$
\left\{\begin{array}{l}
l_{s d} \equiv \sqrt{\frac{\sigma_{\downarrow}\left(1-\beta_{\downarrow}^{2}\right)}{4 e L_{s d}}} \\
\lambda_{s} \equiv \sqrt{\frac{\sigma_{\downarrow}\left(1+\beta_{\downarrow}\right)}{2 e L_{s}}} \\
l_{s f} \equiv \sqrt{\frac{\sigma_{s}\left(1-\beta_{s}^{2}\right)}{4 e L_{s}}} \\
\lambda_{s d} \equiv \sqrt{\frac{\sigma_{s}\left(1-\beta_{s}\right)}{2 e L_{s d}}}
\end{array}\right.
$$

A solution of Eqs. (32) is

$$
\left\{\begin{array}{l}
\Delta \mu_{\downarrow}=\Delta \mu_{1}+\Delta \mu_{2} \\
\Delta \mu_{s}=\lambda_{s}^{2}\left(\left(\frac{1}{l_{s d}^{2}}-\frac{1}{\Lambda_{+}^{2}}\right) \Delta \mu_{1}+\left(\frac{1}{l_{s d}^{2}}-\frac{1}{\Lambda_{-}^{2}}\right) \Delta \mu_{2}\right)
\end{array}\right.
$$


with

$$
\left\{\begin{array}{l}
\Delta \mu_{1}=a_{1} e^{\frac{z}{\Lambda_{+}}}+a_{2} e^{-\frac{z}{\Lambda_{+}}} \\
\Delta \mu_{2}=b_{1} e^{\frac{z}{\Lambda_{-}}}+b_{2} e^{-\frac{z}{\Lambda_{-}}}
\end{array}\right.
$$

where

$$
\Lambda_{ \pm}^{-2}=\frac{1}{2}\left(l_{s d}^{-2}+l_{s f}^{-2}\right)\left(1 \pm \sqrt{1-4 \frac{l_{s d}^{-2} l_{s f}^{-2}-\lambda_{s}^{-2} \lambda_{s d}^{-2}}{\left(l_{s d}^{-2}+l_{s f}^{-2}\right)^{2}}}\right)
$$

The constants $a_{1}, a_{2}, b_{1}, b_{2}$ are defined by the boundary conditions. It can then be seen that the usual spin accumulation corresponding to $\Delta \mu_{s}$ also depends on the spin-conserved $s-d$ electronic diffusion which is known to be efficient [56] and, conversely, that spinconserved diffusion is able to lead to a spin accumulation, or $d$ spin-accumulation effects. Accordingly, we expect to measure some typical effects related to spin-accumulation in single magnetic layers, or if $\beta_{s}=0$ : this point will be illustrated in the new expression of the magnetoresistance (Eq. (39) below), and in Section IV through the effect of current induced magnetization switching (CIMS). $s-d$ relaxation adds a new contribution to the resistance, which plays the role of an interface resistance arising from the diffusive treatment of the band mismatch [64, 65, 66].

The resistance produced by the usual spin-accumulation contribution, plus the contribution of $s-d$ relaxation, are defined (see Eq. (16)) by

$$
R_{s a}=\frac{-1}{e J_{t}} \int_{B}^{A} \frac{\partial}{\partial z}\left(\mu_{i}-\Phi(z)\right) d z
$$

where $\Phi(z)$ is the total electric field and $\mu_{i}$ is one of the chemical potentials. Providing that the total current is $J_{t}=J_{s \uparrow}+J_{s \downarrow}+J_{d \downarrow}$, or

$$
J_{t}=-\frac{\sigma_{t}}{e} \frac{\partial}{\partial z}\left(\frac{\sigma_{d \downarrow}}{\sigma_{t}} \mu_{d \downarrow}+\frac{\sigma_{s \downarrow}}{\sigma_{t}} \mu_{s \downarrow}+\frac{\sigma_{s \uparrow}}{\sigma_{t}} \mu_{s \uparrow}\right)
$$

The total electric field can also be written (from Eqs. (28)) as

$$
\Phi(z)=\frac{J_{t}}{\sigma_{t}}=-\frac{1}{e}\left(\frac{\sigma_{d \downarrow}}{\sigma_{t}} \frac{\partial \mu_{d \downarrow}}{\partial z}+\frac{\sigma_{s \downarrow}}{\sigma_{t}} \frac{\Delta \mu_{\downarrow}}{\partial z}+\frac{\sigma_{s \uparrow}}{\sigma_{t}} \frac{\Delta \mu_{s}}{\partial z}\right)
$$

where $\sigma_{t}=\sigma_{s \uparrow}+\sigma_{s \downarrow}+\sigma_{d \downarrow}$. The resistance is given by :

$$
R_{s a}=-\frac{1}{e J_{t}} \int_{A}^{B}\left(\frac{\sigma_{s \downarrow}}{\sigma_{t}} \frac{\partial \Delta \mu_{\downarrow}}{\partial z}+\frac{\sigma_{s \uparrow}}{\sigma_{t}} \frac{\partial \Delta \mu_{s}}{\partial z}\right) d z
$$

This three-channel model brings to light the interplay between band mismatch effects and spin accumulation, in a diffusive approach. It is interesting to note that the local neutrality 
charge condition which is often used (see for instance Eq. (4) in [87]) was not included, as described in Appendix A. On the contrary, we have imposed the conservation of the current at any point of the conductor. Indeed, electron transfer from a channel to another where the electron mobility is different, induces a local variation of the total current.

The resolution of the coupled diffusion equations is discussed elsewhere [4].

\section{Domain wall scattering}

In the description performed until now, the spin quantification axis that defines up and down spin states was fixed through the whole structure (i.e. through the layers and the interfaces). Providing that the spin quantification axis follows the direction of the magnetization, it could be non-uniform throughout a ferromagnetic layer, or crossing an interface. This is especially the case in the presence of a magnetic domain wall. In a thin enough magnetic domain wall the spin would not follow adiabatically the quantification axis, leading to spin-dependent domain wall scattering (DWS) [60, 61, 62, 63]. This effect has been investigated intensively in the last decades in various structures [69]. The underlaying idea is however rather simple, and can be formulated easily with a generalization of the two-spin channel approach. For the sake of simplicity, this generalization will be performed only for the two electronic populations $\{\alpha, \gamma\}$.

As performed in reference [42] (and appendix B), we start with the conservation of the particles for the two channels, in a discreet model. The system is described by a layer $\Sigma_{k}$ in contact with a left layer $\Sigma_{k-1}$ and a right layer $\Sigma_{k+1}$. The spin-flip scattering introduced in the previous sections is described be the reaction rate $\dot{\Psi}^{k}$. A probability $(1-\Delta \epsilon(k))$ of spinflip alignment along the quantification axis is introduced. In the case of ballistic alignment $(1-\Delta \epsilon(k))=\cos ^{2}(\Delta \theta(k) / 2)$ where $\Delta \theta(k)$ is the angle between the magnetization of two adjacent layers $\Sigma_{k-1}$ and $\Sigma_{k}$. The conservation of the particles is now describes by:

$$
\left\{\begin{array}{l}
\frac{d N_{\alpha}}{d t}=(1-\Delta \epsilon(k)) I_{\alpha}^{k-1 \rightarrow k}-I_{\alpha}^{k \rightarrow k+1}+\Delta \epsilon(k) I_{\gamma}^{k-1 \rightarrow k}-\dot{\Psi}^{k} \\
\frac{d N_{\gamma}}{d t}=(1-\Delta \epsilon(k)) I_{\gamma}^{k-1 \rightarrow k}-I_{\gamma}^{k \rightarrow k+1}+\Delta \epsilon(k) I_{\alpha}^{k-1 \rightarrow k}+\dot{\Psi}^{k}
\end{array}\right.
$$

With the notation introduced in the previous sections, the entropy variation can be written in the following way (Appendix B): 


$$
\begin{aligned}
T \frac{d S}{d t}= & P_{\Phi}^{R^{l} \rightarrow 1}-P_{\Phi}^{\Omega \rightarrow R^{r}} \\
& +\sum_{k=2}^{\Omega} \frac{1}{2}\left(\Delta \mu^{k-1}-\Delta \mu^{k}+2(1-\Delta \epsilon(k)) \Delta \mu^{k}\right) \delta I_{s}^{k-1 \rightarrow k} \\
& +\sum_{k=2}^{\Omega} \frac{1}{2}\left(\mu^{k-1}-\mu^{k}\right) I_{0}^{k-1 \rightarrow k}+\sum_{k=1}^{\Omega} \Delta \mu^{k} \dot{\Psi}^{k}
\end{aligned}
$$

where we have introduced $I_{0}=I_{\alpha}+I_{\gamma}, \delta I=I_{\alpha}-I_{\gamma}, \mu_{0}=\mu_{\alpha}+\mu_{\gamma}$, and $\Delta \mu=\mu_{\alpha}-\mu_{\gamma}$. The terms $P_{\Phi}^{R^{l} \rightarrow 1}$ and $P_{\Phi}^{\Omega \rightarrow 1}$ stand for heat and chemical transfer from the reservoirs to the system $\Sigma$.

After performing the continuum limit, the internal entropy production $\mathcal{I}$ (or irreversibility) reads:

$$
T . \mathcal{I}=-\frac{1}{2} \frac{\partial \mu_{0}}{\partial z} J_{0}+\frac{1}{2}\left(-\frac{\partial \Delta \mu}{\partial z}+2 \epsilon \Delta \mu\right) \delta J+\Delta \mu \dot{\Psi}
$$

The first term is the Joule effect, the second is the dissipation terms related to the spinaccumulation process that occurs at the interface, or for magnetic domain wall, and the third term is the dissipation due to spin-flip (or s-d) electronic relaxation. The expression of the entropy production Eq. (42) allows the Onsager relations generalizing Eq. (11) or Eq. (28) to be deduced:

$$
\left\{\begin{array}{l}
J_{0}=-\frac{\sigma_{0}}{2 e}\left(\frac{\partial \mu_{0}}{\partial z}+\beta\left(\frac{\partial \Delta \mu}{\partial z}-2 \epsilon \Delta \mu\right)\right) \\
\delta J=-\frac{\sigma_{0}}{2 e}\left(\beta \frac{\partial \mu_{0}}{\partial z}+\frac{\partial \Delta \mu}{\partial z}-2 \epsilon \Delta \mu\right) \\
\dot{\Psi}=L_{\alpha \gamma} \Delta \mu
\end{array}\right.
$$

where $\epsilon=\lim _{\Delta k \rightarrow 0} \frac{\Delta \epsilon(k)}{\Delta k}$, and, as already introduced: $\sigma_{0}=\sigma_{\alpha}+\sigma_{\gamma}$ and $\beta=\left(\sigma_{\alpha}-\sigma_{\beta}\right) / \sigma_{t}$.

The diffusion equation for $\Delta \mu$, obtained in the stationary regime, is modified accordingly:

$$
\frac{\partial^{2} \Delta \mu}{\partial z^{2}}=\left(\frac{1}{l_{d i f f}^{2}}+\frac{1}{l_{D W}^{2}}\right) \Delta \mu+\frac{1}{\kappa} \frac{\partial \Delta \mu}{\partial z}
$$

where the length $l_{\text {diff }}$ as been defined in the first section Eq. (20) :

$$
l_{d i f f}=\sqrt{\frac{\sigma_{0}\left(1-\beta^{2}\right)}{2 e L}}
$$


the domain wall diffusion length $l_{D W}$ is defined as:

$$
l_{D W}=\sqrt{\frac{\left(1-\beta^{2}\right)}{4 \epsilon}}
$$

while the length $\kappa$ is given by:

$$
\kappa^{-1}=\epsilon \frac{2 \beta^{2}}{1-\beta^{2}}
$$

The magnetoresistance is modified with respect to Eq. (19), due to the new term $\partial_{z} \Delta \mu(z)$ in the diffusion equation. It is worth pointing out that a spin accumulation $\Delta \mu(z) \neq c s t$ should be expected in case of spin polarized current $(\beta \neq 0)$ even without the usual spin-flip contribution, i.e. in the ballistic limit.

\section{FERROMAGNETIC BROWNIAN MOTION AND MAGNETIZATION SWITCHING}

\section{A. Thermokinetic derivation of the Fokker-Planck equation}

The description performed in the previous sections is related to the transport properties of charge carriers in case of spin polarized current. In spintronics experiments, the electric current is spin-polarized through a ferromagnetic layer, but it is not necessary to describe the ferromagnetic order parameter as such. This is of course no longer the case for current induced magnetization switching experiments, where the magnetization is the measured variable.

The magnetization is a fascinating degree of freedom, that has to be described in length in terms of rotational Brownian motion. The description of the dynamics of ferromagnetic particles coupled to a heat bath is a very active field of investigation [88, 89, 90, 91, 92], and the resulting predictions are rather well known and validated experimentally at large [33, 94, 95] and short (96, 97, 98, 99, 100]) time scales. The magnetization relaxation described here is limited to the so-called Néel relaxation that involves only the magnetic moment, in contrast to the Debye inertial relaxation occurring in ferrofluids (in which the ferromagnetic particles rotates in a viscous environment, leading to surprising inertial effects like negative viscosity [41]).

The aim of this subsection is first to show that the rotational Fokker-Planck equation governing the dynamics of the magnetization $\vec{M}$ of one monodomain particle coupled to the 
heat bath can also be obtained applying step by step the approach used in the previous sections. The resulting Fokker-Planck equation with the corresponding Onsager transport coefficients, and the hypothesis performed, can then be compared term by term to the previous study of spin-dependent charge transport.

\section{Geometrical representation of the statistical ensemble}

Let $\Sigma$ be a statistical ensemble of $N$ identical monodomain particles of volume $v$, having the same energy per unit volume $V^{m a g}(\theta, \phi)$, magnetization $\vec{M}$ and thermostat temperature $T$. The vector $\vec{M}$ is defined by the angles $\theta$ and $\phi$. The ensemble $\Sigma$ can be represented by a distribution of representative points over the unit sphere (fig. [5) with a density $n(\theta, \phi)$.
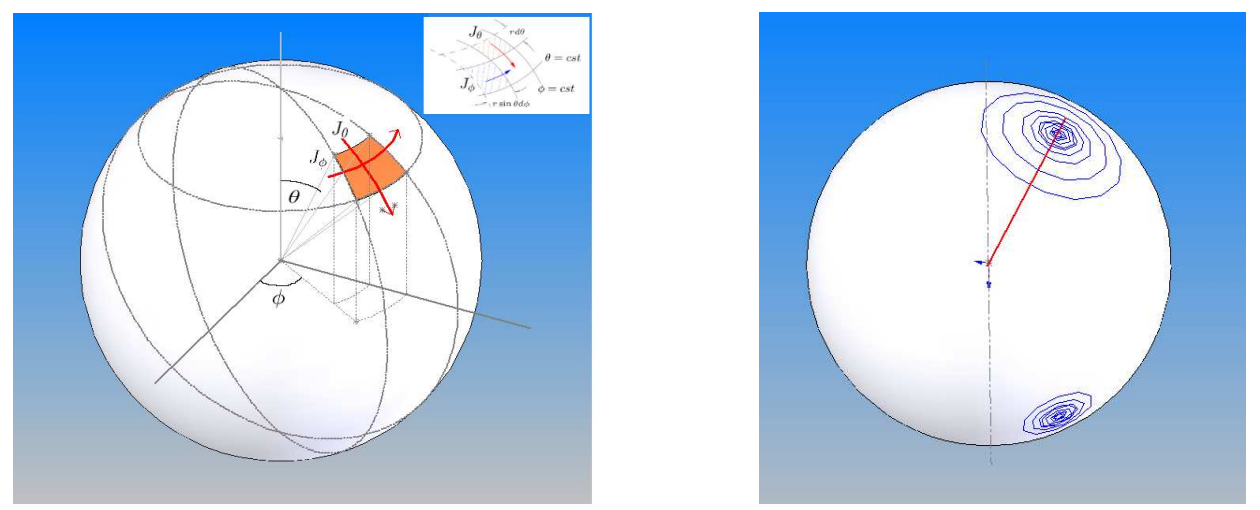

FIG. 5: a) The figure from the left illustrates the flow of representative points over the unit sphere: $J^{\theta}$ and $J^{\phi}$. b) The figure from the right illustrates a particular case of distribution of points on the sphere: the points are concentrated at two attractors, one with more particles than the other (asymmetric double well potential).

We divide the ensemble of representative points $\Sigma$ in sub-ensembles $\Sigma_{\theta, \phi}$ such that the magnetization is confined within the solid angle $\delta V_{\theta, \phi}=\sin \theta d \theta d \phi$. (i.e. the representative points lie between two consecutive parallels and meridians over the sphere).

As the particles undergo changes of magnetization orientation, the representative points move on the sphere, and there is a net surface flux of representative points $\vec{J}^{m a g}$; the representative points move from one sub-ensemble $\Sigma_{\theta, \phi}$ to another sub-ensemble $\Sigma_{\theta+\Delta \theta, \phi+\Delta \phi}$. The probability of finding a particle with the magnetization orientation within the solid angle $d V_{\theta, \phi}$ at a given time $t$ is $d P(\theta, \phi, t)=\frac{n(\theta, \phi, t)}{N} d V_{\theta, \phi}$. 


\section{Conservation laws}

The sub-ensembles of representative points $\Sigma_{\theta, \phi}$ are described by the following extensive parameters: the entropy $d S=s(\theta, \phi, t) d V_{\theta, \phi}$, the number of points $d N=n(\theta, \phi, t) d V_{\theta, \phi}$ and the energy $d E=e(\theta, \phi, t) d V_{\theta, \phi}$, where $s$ and $e$ are the entropy and energy densities. The flow (of points, energy, and entropy) is described by the flux $\vec{J}\left(\vec{J}_{n}, \vec{J}_{e}\right.$ and $\left.\vec{J}_{s}\right)$ :

$$
\vec{J}=J^{\theta} \vec{u}_{\theta}+J^{\phi} \vec{u}_{\phi}
$$

and accounts for the flow of the corresponding magnetic moments relaxing or precessing along the coordinates $\theta$, $\phi$, where $\vec{u}_{r}, \vec{u}_{\theta}, \vec{u}_{\phi}$ are the unit vectors in the spherical coordinate system.

The conservation laws of the number, energy and entropy of the particles contained in the sub-ensemble $\Sigma_{\theta, \phi}$ write :

$$
\left\{\begin{array} { l } 
{ \frac { \partial n } { \partial t } = - \operatorname { d i v } \vec { J } _ { n } } \\
{ \frac { \partial e } { \partial t } = - \operatorname { d i v } \vec { J } _ { e } } \\
{ \frac { \partial s } { \partial t } = - \operatorname { d i v } \vec { J } _ { s } + \mathcal { I } }
\end{array} \Rightarrow \left\{\begin{array}{l}
\frac{\partial n}{\partial t}=-\frac{1}{\sin \theta} \frac{\partial}{\partial \theta}\left(J_{n}^{\theta} \sin \theta\right)-\frac{1}{\sin \theta} \frac{\partial J_{n}^{\phi}}{\partial \phi} \\
\frac{\partial e}{\partial t}=-\frac{1}{\sin \theta} \frac{\partial}{\partial \theta}\left(\mathcal{J}_{e}^{\theta} \sin \theta\right)-\frac{1}{\sin \theta} \frac{\partial \mathcal{J}_{e}^{\phi}}{\partial \phi} \\
\frac{\partial s}{\partial t}=-\frac{1}{\sin \theta} \frac{\partial}{\partial \theta}\left(\mathcal{J}_{s}^{\theta} \sin \theta\right)-\frac{1}{\sin \theta} \frac{\partial \mathcal{J}_{s}^{\phi}}{\partial \phi}+\mathcal{I}
\end{array}\right.\right.
$$

where in contrast to the energy and number of particles, the entropy $s$ is not a conservative quantity, and an internal entropy production term $\mathcal{I}$ (or irreversibility) is added to the entropy flux $\vec{J}_{s}$ (third equation in Eq. (49)).

The expression of the first law of thermodynamics, allows the energy variation to be expressed as a function of the partial derivatives that define the chemical potentials and the temperature $\tilde{\mu} \equiv \frac{\partial e}{\partial n}$ and $T \equiv \frac{\partial e}{\partial s}$. The intensive variables $\tilde{\mu}$ and $T$ are also functions of $(\theta, \phi, t)$ except if imposed by a reservoir.

$$
\frac{\partial s}{\partial t}=\frac{1}{T} \frac{\partial e}{\partial t}-\frac{\tilde{\mu}}{T} \frac{\partial n}{\partial t}
$$

where $\tilde{\mu} \equiv \partial_{n} e$ contains all contributions to the energy (see below)).

The expression of the internal entropy variation can be obtained using the conservation laws:

$$
\frac{\partial s}{\partial t}=-\frac{1}{T} \operatorname{div} \vec{J}_{e}+\frac{\tilde{\mu}}{T} \operatorname{div} \vec{J}_{n}
$$


or, in an other form:

$$
\frac{\partial s}{\partial t}=-\operatorname{div}\left(\frac{1}{T} \vec{J}_{e}-\frac{\tilde{\mu}}{T} \overrightarrow{J_{n}}\right)+\overrightarrow{J_{e}} \cdot \operatorname{grad}\left(\frac{1}{T}\right)-\overrightarrow{J_{n}} \cdot \operatorname{grad}\left(\frac{\tilde{\mu}}{T}\right)
$$

Comparing this last equation with the third eq. from (49), we can deduce the form of the entropy production $\mathcal{I}$ :

$$
\left\{\begin{array}{l}
\overrightarrow{J_{s}}=\frac{1}{T} \vec{J}_{e}-\frac{\tilde{\mu}}{T} \vec{J}_{n} \\
\mathcal{I}=\vec{J}_{e} \cdot \operatorname{grad}\left(\frac{1}{T}\right)-\overrightarrow{J_{n}} \cdot \operatorname{grad}\left(\frac{\tilde{\mu}}{T}\right)
\end{array}\right.
$$

The entropy production $\mathcal{I}$ is a sum of products between the fluxes $\vec{J}_{k}$ and the corresponding conjugate forces $\vec{F}_{k}[38]$.

We assume in the following that the temperature $T(\theta, \phi)=T$ is fixed by a unique thermostat: the first term in the right hand side of the eq. (53) vanishes.

A sufficient condition to impose the second law of thermodynamics $\mathcal{I} \geq 0$ is then to build a quadratic form. This leads us to define the matrix $\mathcal{L}$ of Onsager transport coefficients $L_{i j}(\theta, \phi)$ (that are state functions of dimension $[\text { energy }]^{-1}[\text { time }]^{-1}$ ) such that $J_{i}=\Sigma_{j}\left(L_{i j} \partial_{j} \tilde{\mu}\right)$, where the symmetrized $\mathcal{L}$ matrix is positive. The Onsager reciprocity relations impose furthermore that $L_{i j}= \pm L_{j i}$, where the $\operatorname{sign}(-)$ is present if $L_{i j}$ is a function of the magnetic field (there is no angular velocity here) [35].

The following relations are deduced:

$$
\overrightarrow{J_{n}}=-\mathcal{L} \operatorname{srad} \tilde{\mu} \Rightarrow\left\{\begin{array}{l}
J_{n}^{\theta}=-L_{\theta \theta} \frac{\partial \tilde{\mu}}{\partial \theta}-L_{\theta \phi} \frac{1}{\sin \theta} \frac{\partial \tilde{\mu}}{\partial \phi} \\
J_{n}^{\phi}=-L_{\phi \theta} \frac{\partial \tilde{\mu}}{\partial \theta}-L_{\phi \phi} \frac{1}{\sin \theta} \frac{\partial \tilde{\mu}}{\partial \phi}
\end{array}\right.
$$

where $L_{\theta \phi}=-L_{\phi \theta}$. The first of equations (49) re-writes:

$$
\begin{gathered}
\frac{\partial n_{(\theta, \phi, t)}}{\partial t}=-\operatorname{div} J_{n}=+\operatorname{div}(\vec{L} \operatorname{grad} \tilde{\mu}) \Rightarrow \\
\frac{\partial n_{(\theta, \phi, t)}}{\partial t}=\frac{1}{\sin \theta} \frac{\partial}{\partial \theta}\left[\sin \theta\left(L_{\theta \theta} \frac{\partial \tilde{\mu}_{(\theta, \phi, t)}}{\partial \theta}+\frac{1}{\sin \theta} L_{\theta \phi} \frac{\left.\partial \tilde{\mu}_{(\theta, \phi, t)}\right)}{\partial \phi}\right)\right] \\
+\frac{1}{\sin \theta} \frac{\partial}{\partial \phi}\left[L_{\phi \theta} \frac{\partial \tilde{\mu}_{(\theta, \phi, t)}}{\partial \theta}+\frac{1}{\sin \theta} L_{\phi \phi} \frac{\partial \tilde{\mu}_{(\theta, \phi, t)}}{\partial \phi}\right]
\end{gathered}
$$


where $L_{\theta \theta} \geq 0$ and $L_{\phi \phi} \geq 0$. This is the general expression of the density variation $\partial_{t} n(\theta, \phi, t)$ of particles number from the sub-ensemble $\Sigma_{\theta, \phi}$. Note that there is no relaxation terms $(\dot{\Psi})$ in the conservation law (55) of the magnetization: the flow of representative points is conserved on the unit sphere. This assumption will be removed in the case of spin injection performed with electric currents (see next section below).

In the same manner as for Eq. (12), the Onsager coefficients are related to the relevant relaxation times. In the case of ferromagnetic insulators, the relaxation channels are well defined [101], and the coefficients $L_{i j}$ are directly related to the relaxation times $T_{1}$ and $T_{2}$ measured in ferromagnetic resonance experiments.

\section{B. The rotational Fokker-Planck equation}

In thermokinetics, the intensive parameter which controls the number of particles of a sub-ensemble is the chemical potential $\tilde{\mu}$. The relevant energy terms are contained in the deterministic potential $\mu$, and the stochastic term is defined by thermal fluctuations due to the coupling to a relevant heat bath. Anticipating the last section, it is worth pointing out that the relevant heat bath is defined by the degrees of freedom of the environment which are that of the lattice or that of the electronic system (as discussed below). The fluctuations are taken into account through a temperature dependent chemical potential that takes the following form (derived in the general case by P. Mazur in Ref. [38]) :

$$
\tilde{\mu} \equiv k_{B} T \ln \left(\frac{n}{N}\right)+v V^{m a g}(\theta, \phi)+\mu_{0}
$$

The first term in the right hand side of Eq. (157) is responsible for thermal agitation at temperature $T$, the second term $v V^{m a g}$ represents the magnetic energy of one particle that

defines the local magnetic field $\overrightarrow{H_{e f f}}=-\partial_{\vec{M}}\left(V^{m a g}\right)$ and the third term is a constant which is related to the chemical nature of the particles.

The local equilibrium condition $\partial_{i} \tilde{\mu}=0$ defines stationary flux (due to both drift and diffusion) that are mutually compensated along the coordinate $i$. This point is well illustrated in the work of Guggenheim while introducing the electro-chemical potential [33] in order to generalize the description of an electric fluid to ionic solutions.

Inserting the expression of $\tilde{\mu}$, and using the reciprocal relation $L_{\theta \phi}=-L_{\phi \theta}$, the equations for fluxes and the variation of particles take the form: 


$$
\left\{\begin{array}{l}
J_{n}^{\theta}=-\left(h^{\prime} \frac{\partial V^{m a g}}{\partial \theta}-\frac{g^{\prime}}{\sin \theta} \frac{\partial V^{m a g}}{\partial \phi}\right) n-\left(h^{\prime} \frac{k_{B} T}{v} \frac{\partial n}{\partial \theta}-\frac{g^{\prime}}{\sin \theta} \frac{k_{B} T}{v} \frac{\partial n}{\partial \phi}\right) \\
J_{n}^{\phi}=-\left(g^{\prime} \frac{\partial V^{m a g}}{\partial \theta}+\frac{k^{\prime}}{\sin \theta} \frac{v}{k_{B} T} \frac{\partial V^{m a g}}{\partial \phi}\right) n-\left(g^{\prime} \frac{k_{B} T}{v} \frac{\partial n}{\partial \theta}+\frac{k^{\prime}}{\sin \theta} \frac{\partial n}{\partial \phi}\right)
\end{array}\right.
$$

where the following standard notations have been introduced:

$$
h^{\prime}=\frac{L_{\theta \theta} v}{n} \geq 0 ; \quad g^{\prime}=-\frac{L_{\theta \phi} v}{n}=\frac{L_{\phi \theta} v}{n} ; \quad k^{\prime}=L_{\phi \phi} \frac{k_{B} T}{n} \geq 0
$$

Assuming that $g^{\prime}$ is constant, Equation Eq. (56) rewrites:

$$
\begin{aligned}
\frac{\partial n(\theta, \phi)}{\partial t}=\frac{1}{\sin \theta} \frac{\partial}{\partial \theta}\{\sin \theta & {\left.\left[\left(h^{\prime} \frac{\partial V^{m a g}}{\partial \theta}-\frac{g^{\prime}}{\sin \theta} \frac{\partial V^{m a g}}{\partial \phi}\right) n+h^{\prime} \frac{k_{B} T}{v} \frac{\partial n}{\partial \theta}\right]\right\} } \\
+ & \frac{1}{\sin \theta} \frac{\partial}{\partial \phi}\left\{\left(g^{\prime} \frac{\partial V^{m a g}}{\partial \theta}+\frac{k^{\prime}}{\sin \theta} \frac{v}{k_{B} T} \frac{\partial V^{m a g}}{\partial \phi}\right) n+\frac{k^{\prime}}{\sin \theta} \frac{\partial n}{\partial \phi}\right\}
\end{aligned}
$$

The expression Eq. (60) represents the rotational Fokker-Planck equation obtained by thermokinetic means. Its expression is identical to that obtained by Brown [89] through stochastic calculations.

Furthermore, the Onsager matrix also follows the symmetry of the system, and is invariant by rotation around the anisotropy axis, so that $: L_{\phi, \phi}=L_{\theta, \theta}$. The following relations are obtained :

$$
L_{\theta \theta}=L_{\phi \phi}=\frac{h^{\prime} n}{v}=\frac{k^{\prime} n}{k_{B} T}
$$

which permits us to write the equations above in a compact vectorial form:

$$
\left\{\begin{aligned}
\vec{J} & =-g^{\prime} \vec{u}_{r} \times\left(n \vec{\nabla} V^{m a g}+\frac{k_{B} T}{v} \vec{\nabla} n\right)+h^{\prime} \vec{u}_{r} \times\left[\vec{u}_{r} \times\left(n \vec{\nabla} V^{m a g}+\frac{k_{B} T}{v} \vec{\nabla} n\right)\right] \\
\frac{\partial n}{\partial t} & =g^{\prime} \vec{\nabla}\left\{\vec{u}_{r} \times\left(n \vec{\nabla} V^{m a g}+\frac{k_{B} T}{v} \vec{\nabla} n\right)\right\}-h^{\prime} \vec{\nabla}\left\{\vec{u}_{r} \times\left[\vec{u}_{r} \times\left(n \vec{\nabla} V^{m a g}+\frac{k_{B} T}{v} \vec{\nabla} n\right)\right]\right\}
\end{aligned}\right.
$$

where the gradient $\vec{\nabla}$ is in spherical coordinates and $\vec{u}_{r}$ is the spherical radial unit vector. It is to be noticed that the second equation has drift terms which contain $\nabla V^{m a g}$, and diffusion terms which contain $\vec{\nabla} n$. The terms $k^{\prime}=h^{\prime} \frac{k_{B} T}{v}$ and $g^{\prime} \frac{k_{B} T}{v}$ are the rotational diffusion coefficients and the terms $g^{\prime} n$ and $h^{\prime} n$ represent drift coefficients. 


\section{Landau-Lifshitz-Gilbert equation with diffusion}

Furthermore, using the first equation from (62), one can deduce the Landau-LifshitzGilbert equation with diffusion. As $\vec{J}=n \frac{d \vec{u}_{r}}{d t}$, we arrive at the equation:

$$
\frac{d \vec{u}_{r}}{d t}=-g^{\prime} \vec{u}_{r} \times\left(\vec{\nabla} V^{m a g}+\frac{k_{B} T}{v} \frac{\vec{\nabla} n}{n}\right)+h^{\prime} \vec{u}_{r} \times\left[\vec{u}_{r} \times\left(\vec{\nabla} V^{m a g}+\frac{k_{B} T}{v} \frac{\vec{\nabla} n}{n}\right)\right]
$$

where the first term in the right hand side is the precession term, and the second term in the right hand side describes the longitudinal relaxation. Multiplied by the amplitude of the magnetization $M_{s}$, becomes the Landau-Lifshitz-Gilbert equation with diffusive terms:

$$
\frac{d \vec{M}}{d t}=g^{\prime} M_{s} \vec{M} \times\left(\overrightarrow{H_{e f f}}-\frac{k_{B} T}{v M_{s}} \frac{\vec{\nabla} n}{n}\right)+h^{\prime}\left[\vec{M} \times\left(\overrightarrow{H_{e f f}}-\frac{k_{B} T}{v M_{s}} \frac{\vec{\nabla} n}{n}\right)\right] \times \vec{M}
$$

Experimentally, the first contribution can be observed through ferromagnetic resonance measurements (FMR) at typical frequencies of tens of $\mathrm{GHz}$ (around 100 psec time scales). The thermalization time (proportional to $1 / \mathrm{g}$ ' ; see next section) is given by the width of the resonance peaks. Both frequency and time resolved noise experiments have been also performed in order to measure precession and thermal spin-waves [96, 97, 98, 99, 100]. In the above measurements, the data are averaged over many shots (or trajectories) near an equilibrium position (linear response regime). In contrast, the measurements at large time scales, typically beyond few nanosecond, access the magnetization reversal for which the precession terms can be neglected. The one shot measurements give a direct access to the stochastic nature of the signal [21, 22, 25, 93, 94]: a snapshot is a statistical event, namely the magnetization reversal from one metastable state to the other, that is governed by the random fluctuations, described by a "Langevin force" that is not present in the averaged LLG equation.

Equation Eq. (63) can be put into the Gilbert form by performing the cross product $\times \vec{M}$ at the left and right hand side of the equation. We obtain the well-known Gilbert equation, that defines the Gilbert damping parameter $\eta$ :

$$
\frac{d \vec{M}}{d t}=\Gamma \vec{M} \times\left(\vec{H}_{e f f}-\eta \frac{d \vec{M}}{d t}\right)
$$


where $\Gamma$ is the gyromagnetic factor. The constant h', g' and k' are related to the Gilbert damping coefficient $\eta, \Gamma$ and the magnetization at saturation $M_{s}$ through the following relations:

$$
g^{\prime}=\frac{\Gamma}{\left(1+\left(\eta \Gamma M_{s}\right)^{2}\right) M_{s}} \geq 0 ; \quad h^{\prime}=\eta \Gamma M_{s} g^{\prime}
$$

or

$$
\eta=\frac{1}{\Gamma M_{S}} \sqrt{\frac{\Gamma}{g^{\prime} M_{s}}-1} \geq 0
$$

\section{Activation regime and Néel-Brown law}

\section{Neglecting precession}

In the slow relaxation measurements (the so called magnetic after-effect), relaxation is governed by activation over a potential barrier. At longtime scales (beyond tens of nanosecond to hour), the precessional terms can be neglected. The expression for the surface current fluxes Eq. (58) becomes

$$
\left\{\begin{array}{l}
J_{n}^{\theta}=-h^{\prime} \frac{\partial V^{m a g}}{\partial \theta} n-h^{\prime} \frac{k_{B} T}{v} \frac{\partial n}{\partial \theta} \\
J_{n}^{\phi}=-g^{\prime} \frac{\partial V^{m a g}}{\partial \theta} n-g^{\prime} \frac{k_{B} T}{v} \frac{\partial n}{\partial \theta}
\end{array}\right.
$$

and the corresponding Fokker-Planck equation becomes:

$$
\frac{\partial n(\theta, \phi)}{\partial t}=\frac{1}{\sin \theta} \frac{\partial}{\partial \theta}\left\{\sin \theta\left[h^{\prime} \frac{\partial V^{m a g}}{\partial \theta} n+h^{\prime} \frac{k_{B} T}{v} \frac{\partial n}{\partial \theta}\right]\right\}
$$

These expressions will be used in the following paragraph for deriving the Néel-Brown relaxation time.

\section{The double-well potential and the relaxation times}

The double well potential (see fig. 6) is the first approximation of the ferromagnetic particle energy $V^{\operatorname{mag}}(\theta, \phi)$ beyond the harmonic potential, but it is also a realistic magnetic potential in the case of an uniform magnetization with uniaxial anisotropy [22]:

$$
V^{m a g}=K \sin ^{2} \theta-M_{s} H_{a p} \cos (\theta-\varphi)
$$


where $\theta$ is the direction of the magnetization, $\varphi$ the direction of the applied field $H_{a p}$, and $\mathrm{K}$ is the anisotropy constant in energy per unit volume.

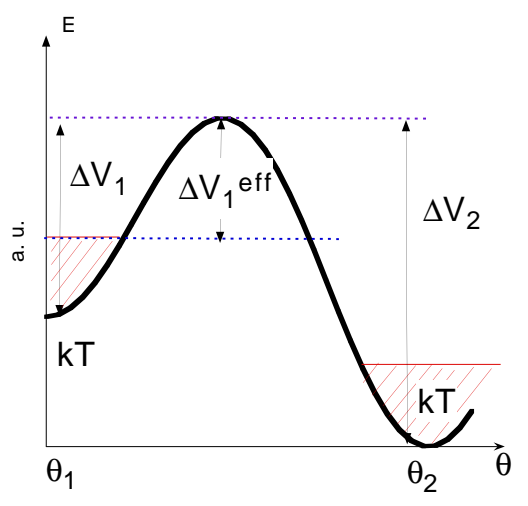

FIG. 6: Double well potential (continuous line) with stochastic fluctuations (dashed area), and the definition of the barrier heights.

In order to apply Brown's method, we consider a potential which has minima at $\theta_{1}$ and $\theta_{2}=\pi-\theta_{1}$, and a maximum at $\theta_{m}$. Following Kramers transition theory, Brown [89] assumes that most of the representative points on the unit sphere are concentrated at the energy minima of $V^{m a g}(\theta)$ where they are in thermal equilibrium so that locally $n$ takes the form of the Maxwell-Boltzmann distribution. Thus only a minute fraction of the representative points is outside the energy minima allowing a small diffusion current between them so manifesting the non-equilibrium conditions.

$$
n(\theta, \phi)= \begin{cases}n\left(\theta_{1}\right) e^{-\frac{v}{k_{B} T}\left[V(\theta)-V\left(\theta_{1}\right)\right]}, & \text { for } \theta \in\left(\theta_{1}-\epsilon, \theta_{1}+\epsilon\right) \\ n\left(\theta_{2}\right) e^{-\frac{v}{k_{B} T}\left[V(\theta)-V\left(\theta_{2}\right)\right]}, & \text { for } \theta \in\left(\theta_{2}-\epsilon, \theta_{2}+\epsilon\right)\end{cases}
$$

The number of particles $N_{1} \leftrightarrow N_{2}$ from the first well, respectively the second is:

$$
\left\{\begin{array}{lll}
N_{1}=2 \pi n\left(\theta_{1}\right) e^{\frac{v}{k_{B} T} V\left(\theta_{1}\right)} I_{1}, & \text { where } & I_{1}=\int_{\theta_{1}-\epsilon}^{\theta_{1}+\epsilon} e^{-\frac{v}{k_{B} T} V(\theta)} \sin \theta d \theta \\
N_{2}=2 \pi n\left(\theta_{2}\right) e^{\frac{v}{k_{B} T} V\left(\theta_{2}\right)} I_{2}, & \text { where } & I_{2}=\int_{\theta_{2}-\epsilon}^{\theta_{2}+\epsilon} e^{-\frac{v}{k_{B} T} V(\theta)} \sin \theta d \theta
\end{array}\right.
$$

Assuming that the flow between the two minima $\theta_{1}$ and $\theta_{2}$ is quasistationary, approximated by a divergenceless current [89], the total current of particles over the potential barrier can be written:

$$
I=2 \pi \sin \theta J_{n}^{\theta}
$$


Rewriting the first equation from 68, one obtains:

$$
\frac{\partial n}{\partial \theta}+\frac{v}{k T} \frac{\partial V^{m a g}}{\partial \theta} n=-\frac{I v}{2 \pi h^{\prime} k_{B} T \sin \theta}
$$

which defines the activation regime. Introducing $I_{m}$ as

$$
I_{m}=\int_{\theta_{1}+\Delta \epsilon}^{\theta_{2}-\Delta \epsilon} \frac{e^{\frac{v}{k_{B} T} V(\theta)}}{\sin \theta} d \theta
$$

Eq. (74) yields

$$
I=-\dot{N}_{1}=\dot{N}_{2}=-\frac{h^{\prime} k_{B} T}{v I_{m}}\left(\frac{N_{2}}{I_{2}}-\frac{N_{1}}{I_{1}}\right)
$$

which has the form

$$
\dot{N}_{1}=-\dot{N}_{2}=\frac{N_{2}}{\tau_{2}}-\frac{N_{1}}{\tau_{1}}
$$

with

$$
\left\{\begin{array}{l}
\tau_{1}=\frac{I_{1} I_{m} v}{h^{\prime} k_{B} T} \\
\tau_{2}=\frac{I_{2} I_{m} v}{h^{\prime} k_{B} T}
\end{array}\right.
$$

Because of the rapid decrease of the exponential factor with distance from the minima of $V^{m a g}$, we may in $I_{1}, I_{2}, I_{m}$ replace $V^{m a g}(\theta)$ by its Taylors's series about $\theta_{1}, \theta_{2}$, respectively $\theta_{m}$ truncated at the $\theta_{2}$ term, and replace the upper limit of the integrals by $\infty$. With these approximations, we find

$$
\left\{\begin{array}{l}
\tau_{1}=\tau_{01} e^{\frac{v\left(V\left(\theta_{m}\right)-V\left(\theta_{1}\right)\right)}{k_{B} T}} \\
\tau_{2}=\tau_{02} e^{\frac{v\left(V\left(\theta_{m}\right)-V\left(\theta_{2}\right)\right)}{k_{B} T}}
\end{array}\right.
$$

where the waiting times are given by the expressions:

$$
\left\{\begin{array}{l}
\tau_{01}=\frac{2 \pi}{h^{\prime}}\left[-V^{\prime \prime}\left(\theta_{1}\right) V^{\prime \prime}\left(\theta_{m}\right)\right]^{-\frac{1}{2}} \frac{\sin \theta_{1}}{\sin \theta_{m}} \\
\tau_{02}=\frac{2 \pi}{h^{\prime}}\left[-V^{\prime \prime}\left(\theta_{2}\right) V^{\prime \prime}\left(\theta_{m}\right)\right]^{-\frac{1}{2}} \frac{\sin \theta_{2}}{\sin \theta_{m}}
\end{array}\right.
$$

Equation Eq. (79) is a formula for a symmetric bistable potential which has minima in $\theta_{1}, \theta_{2}=\pi-\theta_{1}$ and a maximum in $\theta_{m}=\pi / 2$.

For $\varphi \neq 0$, the potential $V^{m a g}(\theta, 0)$ has an asymmetric bistable form, and all arguments leading to Eq. (79) also apply for an arbitrary $\varphi$ [91]. The general equations are very similar 
to Eq. (79), the only difference in the analytic expression is that instead of the symmetric angles $\theta_{1}, \theta_{2}$, we have the asymmetric angles $\theta_{A}$, respectively $\theta_{B}$.

It has to be emphasized that $\tau_{1}$ and $\tau_{2}$ are the relaxation times related to the first and second potential barrier (starting from the first or the second minima), and that Eq. (79) constitutes the Néel-Brown law for the particular case of the symmetric bistable well. Interestingly, in all cases [91], the Gilbert damping is reduced to the prefactor of the exponential, and consequently plays only a negligible role in the activation process.

In many cases, the potential is highly asymmetric, and the Néel-Brown law is written in the following asymptotic form:

$$
\tau=\tau_{0} \exp \left(\frac{\Delta V_{0}\left(1-H / H_{s w}^{0}\right)^{\alpha}}{k T}\right)
$$

with three phenomenological parameters $\alpha \approx 3 / 2, \Delta V_{0}$ and $H_{s w}^{0}$ [93, 94]. The laws (79) and (81) are well established experimentally in usual magnetic sub-micro structures. More surprisingly, they have been also observed in CIMS experiments under spin-injection with high effective temperature $T_{\text {eff }}(2000 \mathrm{~K}$ to $20000 \mathrm{~K}$ ) instead of $300 \mathrm{~K}$ to $340 \mathrm{~K}$ 2, 21, 22, 23, 24, 25] (see next section).

Fig. 7 describes how to measure the ferromagnetic potential landscape with the help of slow magnetic relaxation measurements (the so called magnetic after-effect measurements), performed on a single magnetic domain nanostructure 22]. In the example shown, the angle and amplitude of the magnetic field is set in order to obtained the two-level fluctuation effect (measurements reported in Wernsdorfer et al. [94]). The principle of the measurements is sketched in Fig. 7 for a sample with uniaxial anisotropy : the hysteresis loop describes the succession of equilibrium magnetization configurations (or quasi-static states) as a function of the magnetic field (the field is normalized to the anisotropy) for different angles $\varphi$ of the applied field. The angle $\theta$ describes the direction of the magnetization. For a given angle of the field $\varphi$, the hysteresis is composed of the reversible configurations and two symmetric irreversible jumps over the potential barrier. The jump occurs from one equilibrium (metastable) state defined by the angle $\theta_{1}$ to the other, defined by the angle $\theta_{2}$ (Fig. Z (b)). At zero Kelvin (without fluctuation), this angle is related to the applied field through a relation that depends on the switching mode (the switching mode concerns the magnetic configurations occurring during the jump, i.e. the modes with a typical lifetime of fraction of nanoseconds [89, 102]). In contrast, the amplitude of the jump is easily measured (it 
is given by the difference $\left.M_{s}\left(\cos \theta_{2}-\cos \theta_{1}\right)\right)$, and gives direct access, from the hysteresis loop, to the quasi-static configurations (i.e. to the position of the potential wells). Changing the field (amplitude and angle) necessarily changes the equilibrium position (defined by $\nabla V(H)=0)$ and the angles $\theta_{1}$ and $\theta_{2}$. Note that the case of uniaxial symmetry with $\varphi=0$ is the unique pathological case, where the change in the amplitude of the field does not change the equilibrium magnetic configurations. However, since this pathological case is unfortunately that used in most calculations for the sake of simplicity (this was also the case here for the derivation of the Néel-Brown's law), the fact the initial and final states are necessarily modified is not mentioned in many reports of ferromagnetic relaxation experiments. Inversely, a change observed in the two equilibrium configurations implies a change of the potential landscape, i.e. a change of the effective field.

The possibility of distinguishing between the effect of an effective field and the action of the environment will be of fundamental importance with regard to the study of magnetization reversal due to spin injection (that are performed on single domain magnetic nanostructures), because the main problem is to identify the typical relaxation times that govern the mechanisms responsible for the magnetization reversal (effective fields vs. stochastic fluctuations). It is worth pointing out that it is not relevant (and sometimes misleading) to measure relaxation effects in order to access the valley of the potential (i.e. the reversible magnetic configurations induced by the action of an effective field) because the position of the valley is given by the quasi-static measurements (the hysteresis loop). Furthermore, the stochastic fluctuations do not perturb the quasi-static configurations (e.g. reversible part of the hysteresis), except at the two critical points where the potential barrier is of the same order as the thermal energy. In other terms, only the position of the irreversible jump is modified by the activation (or by the observation time window, or equivalently by the velocity of the sweeping field [103]), but not the rest of the hysteresis loop. Of course, this is no longer the case for larger samples, beyond nanoscopic dimensions, because the sample is a distribution of single domain sub-systems with a distribution of anisotropies, defects, etc. In contrast, the barrier height - or the amplitude of the thermal fluctuations - can be measured only by the activation process, through statistical measurements. If the barrier height is above the energy of the lattice $k T$, the two level fluctuations occur within a typical time window that can be tuned with the amplitude of the magnetic field or the temperature. In order to access to the relaxation times, statistics should be performed over a significant 
number of shots. If the exponential relaxation is verified, the relaxation times can then be extracted. The ratio of the relaxation times $\tau\left(\theta_{1}\right) / \tau\left(\theta_{2}\right)$ gives the asymmetry of the double well, and each relaxation time gives the corresponding barrier height. The Néel-Brown law is tested by varying the temperature and the magnetic field (Fig. 7). The whole potential can be rebuilt form these measurements.

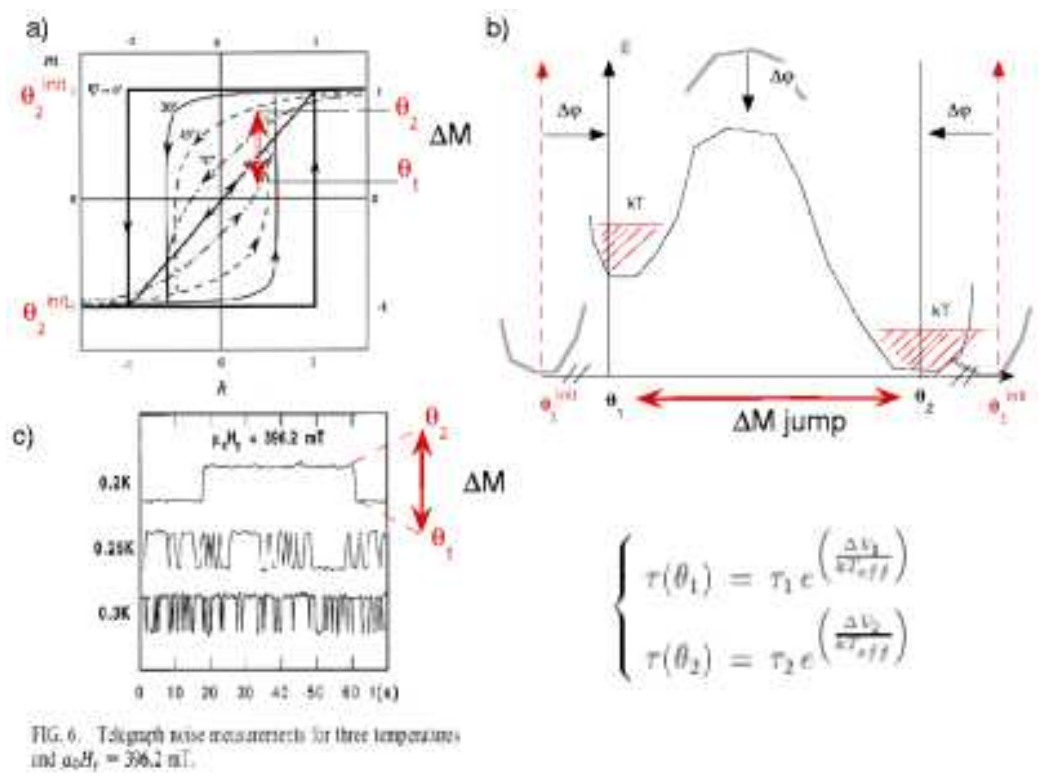

FIG. 7: Slow ferromagnetic relaxation measurements in uniaxial anisotropic single magnetic domain. (a) hysteresis loop at different angles $\varphi$ of the applied magnetic field. The saturation (i.e. initial states without excitation in the relaxation protocol: $H_{y}=0$ ) corresponds to the magnetization $\theta_{1}^{\text {init }}$ or $\theta_{2}^{\text {init }}$. (b) double well potential after the application of the excitation $\Delta \varphi$ : the whole potential landscape is modified (the barrier height and the two equilibrium configurations). (c) two level fluctuations measured between the two configurations (the magnetization jumps from potential well at $\theta_{1}$ to the potential well at $\theta_{2}$. Right: the Néel-Brown law is verify after performing statistics over a significant number of jumps, in order to access to the mean relaxation times $\tau\left(\theta_{1}\right)$ and $\tau\left(\theta_{2}\right)$. Reprint with the permission from Ref. 94] Phys. Rev. Lett. 78, 1791 (1997), Copyright@American Physical Society 


\section{SPIN-INJECTION INDUCED FERROMAGNETIC BROWNIAN MOTION}

Let us now consider a system composed of a ferromagnet in which spin-injection (with high current density) is performed. In such spin-transfer experiments, the magnetization configurations are measured while injecting the current. Usually the magnetic configurations are measured with GMR or AMR properties for convenience, but micro-magnetometry or magneto-optics measurements are also possible [104]. The magnetization is a macroscopic (thought nanoscopic) variable described in the previous section (Sec. III), and the spininjection is described by the two -or four- channel model presented in section II. We start with the assumption that the magnetic system is an open system, composed on one hand of the spin-accumulated charge carriers, and on the other hand by the ferromagnetic order parameter. It is worth pointing out that in this picture, we are not dealing with a theory of itinerant ferromagnets, in which spin injection is performed. Such a theory would allow the dynamics of the macroscopic variable $\vec{M}$ to be derived from the spin-dependent electronic populations $\delta n$ (e.g. a theory in which the LLG equation would be derived from the Hamiltonian of the electronic system [105]). Such a theory would be very difficult because statistical projections should necessarily be performed in order to reduce the microscopic degrees of freedom to fluctuations and damping for the macroscopic order parameter 106, 107, 108, 109]. The simple phenomenological model proposed here can be viewed as a first step in this direction, by defining the different sub-systems, that are coupled through relaxation processes (see first Figure in Sec. I).

\section{A. Current-dependent effective field?}

Before dealing with the dissipative coupling invoked above, a first trivial mechanism that couples the two sub-systems should be discussed. This is a deterministic coupling through a common electro-magneto-chemical potential $\mu$, from which the associated effective fields are derived $\partial_{x} \mu_{0}, \partial_{x} \Delta \mu$ (electric) $\partial_{\theta} \mu$ (magnetic), $\Delta \mu$ (spin pumping). This concept follows that introduced by Guggenheim [33] when he defined the electro-chemical potential in order to describe electrochemical or electrophoretic processes. For instance, the local electric field $\partial_{x} \Delta \mu$ is spin-dependent, and might have an action on the magnetic order parameter. On the other hand, the magnetic field $\partial_{\vec{u}} V^{m a g}$ is able to have an action on the spin polarized 
current.

These effects are defined by the deterministic part $\mu$ of the chemical potential $\tilde{\mu}$ and can hence be reduced to the action of the effective field $\vec{H}_{e f f}(I)=-\vec{\nabla} \mu$ (drift part of the Fokker-Planck equation, to be opposed to the diffusion part), according to Eq. (60). In other terms, within this hypothesis, CIMS experiments reproduce the slow magnetic relaxation (or magnetic after-effect) experiments described in the last paragraph of the previous section, where the magnetic field excitation is replaced by the injection of current.

In the context of thermokinetics, the two mechanisms are identical: the current or the field drives the magnetization from a stable initial position $\left(\theta^{\text {init }}\right)$ to the metastable state $\theta_{1}$ or $\theta_{2}$ by modifying the position of the potential landscape. If a relaxation is observed, this is due to the usual activation process only, i.e. the jump over the barrier induced by the thermal fluctuation (helped by the Joule heating due to the current) [110, 111, 112]. Accordingly, as underlined at the end of the previous section, this deterministic mechanism due to current injection should first be observed on the stable magnetic configurations, by measuring the modification of the potential wells (e.g. measuring the modification of the hysteresis loop of a single domain nanolayer) under current injection.

However, it is worth pointing out that the modification of the potential landscape is not observed: the basic prediction of the determinist action of the spin-injection has not been verified until now [1, 2, 4, 21, 22, 23, 25]. Fig. 8 shows two shots with two level fluctuations in a time interval of milliseconds (a) ([21]) and microseconds (b) ([23]), obtained by different groups with GMR measurements on trilayer nanopillars, and single irreversible jumps measured in an hysteresis loop of a Ni nanowires (c) (25]): each point in the zoom (right part of Fig. 8 (c)) is a 6 microseconds pulse. The hysteresis is performed with current varying from $2.410^{7} \mathrm{~A} / \mathrm{cm}^{2}$ to $1.510^{7} \mathrm{~A} / \mathrm{cm}^{2}$ (the position of the jump without current injected is shown by the arrow). In all cases, the metastable states (described by the angles $\theta_{1}$ and $\theta_{2}$ defined in the previous section) coincides with $\theta_{1}^{\text {init }}$ and $\theta_{2}^{\text {init }}$ measured before the application of the current. All happens as if the potential landscape were not modified!

In contrast, the action of the current is huge (some fraction of an $\mathrm{eV}$ to few $\mathrm{eV}$, i.e. from $25 \%$ to more than $100 \%$ of the anisotropy energy of the ferromagnet), and is observed only for the irreversible jump. By acting on the jump and not on the potential landscape, the effect of the current mimics the action of a temperature.

The arguments developped above are based on an analysis performed with neglecting 

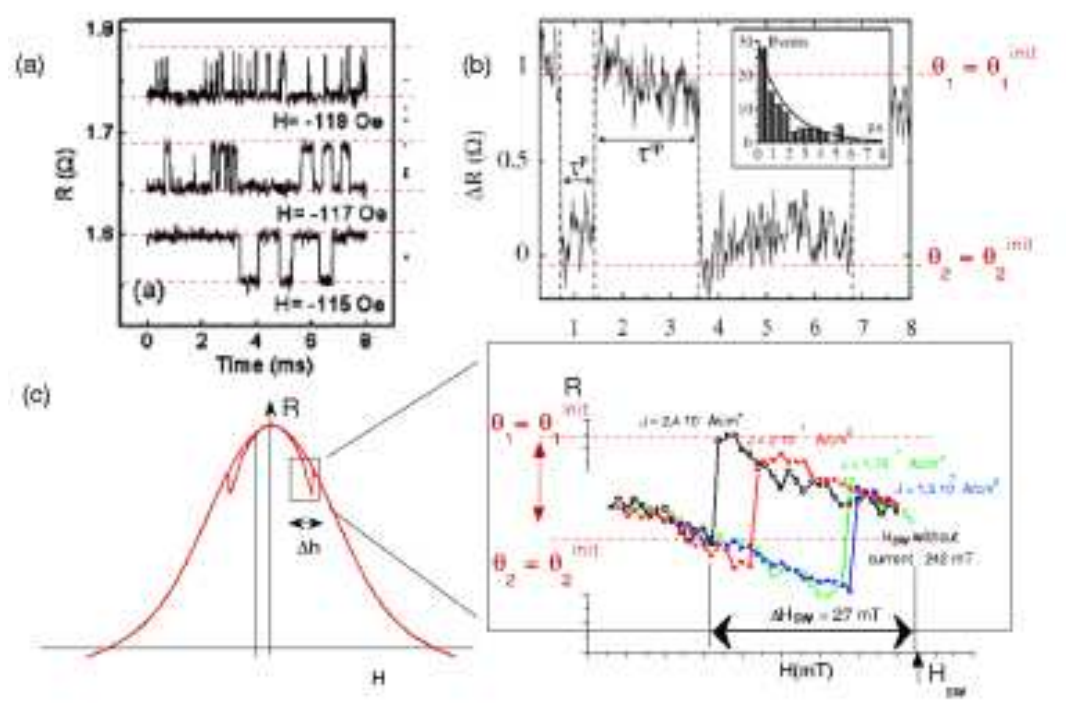

FIG. 8: Ferromagnetic relaxation measurements under spin-injection. (a) Two level fluctuation measured with GMR in a trilayer system (Permaloy/Cu/Permaloy, from [21]). (b) Two level fluctuations measured with GMR in a trilayer structure $(\mathrm{Co} / \mathrm{Cu} / \mathrm{Co}$, from [23]). (c) Hysteresis loop measured with AMR (left) and zoom around the irreversible jump measured under spininjection (6 microseconds pulse per point) (details reported in 25]). The hysteresis measured with AMR shows the succession of the equilibrium configurations. In all cases, the initial and final states (the equilibrium states) are that measured without current injection $\left(\theta=\theta^{\text {init }}\right)$. Equilibrium configurations are not modified by the spin-injection. Reprint with the permission from Ref. 21] S. Uhrazdhin, et al. Phys. Rev. Lett. 91146803 (2003) and A. Fabian, et al. Phys. Rev. Lett. 91 257209 (2003), Copyright@American Physical Society.

the precession terms. If we assume an hypothetical precession maintained in a stationary regime, the magnetization is then driven by the precession, and the trace in Fig. 6 should be interpreted as trajectories in the phase space. Within this context, the precession induces intermittency, i.e. a highly specific chaotic behavior in which the time spent out of the attractors is negligible. Such an approach is discussed in some theoretical works about CIMS. This interpretation is however evacuated here because the measured traces (Fig. 6) mimic exactly the full stochastic process composed of the two-level fluctuation (that follows the Néel-Brown activation law), superimposed to the same white noise for both quasi-static states 113]. The observation of identical noise in both states is in contradiction with published 
simulations performed with the hypothesis of spin transfer torque [114, 115]. Furthermore, after spending years in measuring activation processes in ferromagnetic nanostructures, it is difficult to accept that a deterministic behavior replaces the activation and mimics so perfectly the full stochastic behavior.

Accordingly, we focus our attention on the dissipative, or irreversible, spin-transfer effect, for which the action of the current is expressed through the diffusive terms of the FokkerPlanck equation .

\section{B. Negative damping}

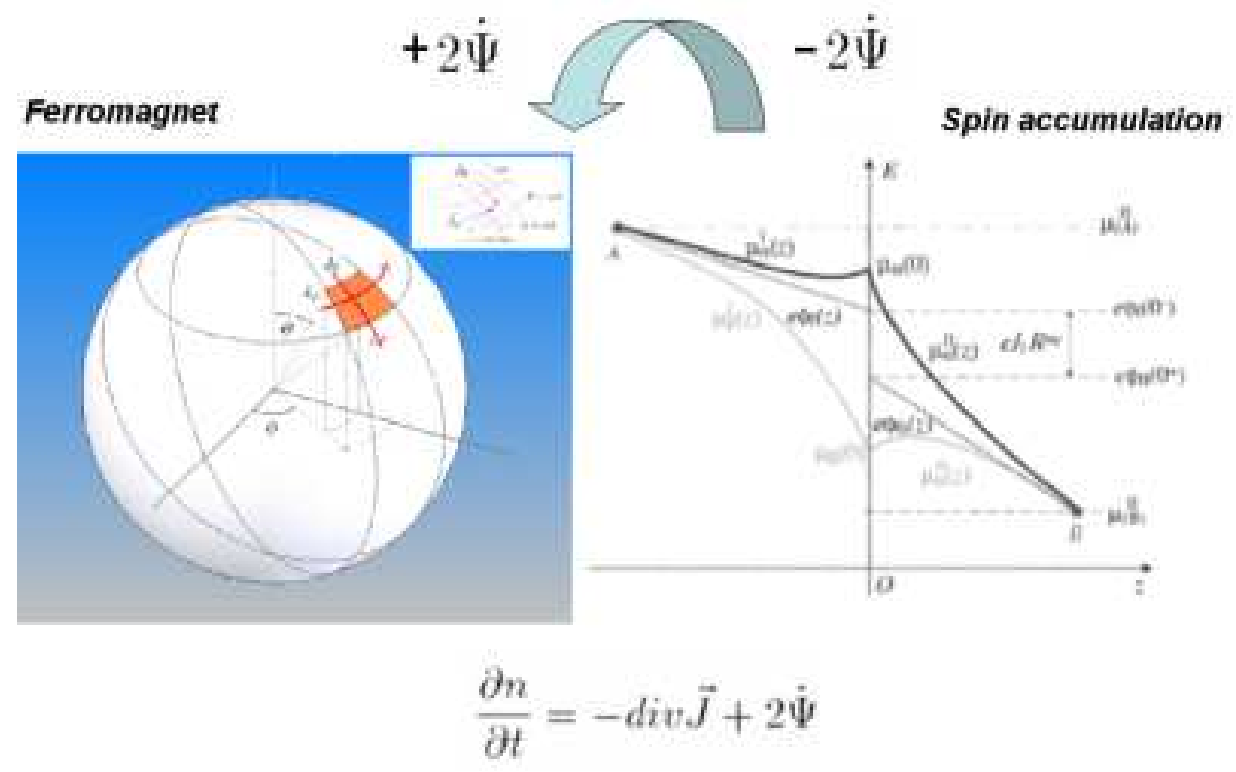

FIG. 9: Under spin-injection, the ferromagnetic layer is an open system coupled to the spinaccumulation reservoir. The variation of the density of magnetic moments (oriented at a given direction in the unit sphere) is given by the divergence of the magnetic flux added the correction due to the relaxation rate $\dot{\Psi}$ of spins relaxing from one system to the other at the interfaces.

In order to describe the ferromagnetic layer coupled to the spin-dependent electric subsystem, we rewrite the conservation law of the ferromagnetic systems (Eq. [55) with adding the relaxation term $\dot{\Psi}$ due to spin-transfer, while substracting it from the spin-accumulation's conservation law: 


$$
\left\{\begin{array}{c}
\frac{\partial n^{\text {ferro }}}{\partial t}=-\operatorname{div}\left(\overrightarrow{J_{n}}\right)+\dot{\Psi} \\
\frac{\partial n^{e l e c}}{\partial t}=-\operatorname{div}(\overrightarrow{\delta J})-\dot{\Psi}
\end{array}\right.
$$

According to the first law of thermodynamics, the contribution due to the electronic relaxation $\dot{\Psi}$ should be taken into account in the entropy production of the ferromagnet. Inserting Eq. (82) into Eq. (51), the entropy production is:

$$
\left\{\begin{array}{l}
\overrightarrow{J_{s}}=\frac{1}{T} \overrightarrow{J_{e}}-\frac{\tilde{\mu}}{T} \overrightarrow{J_{n}} \\
\mathcal{I}=\overrightarrow{J_{e}} \cdot \operatorname{grad}\left(\frac{1}{T}\right)-\overrightarrow{J_{n}} \cdot \operatorname{grad}\left(\frac{\tilde{\mu}}{T}\right)+\frac{\Delta \mu}{T} \dot{\Psi}
\end{array}\right.
$$

where $\Delta \mu=\mu_{\text {elec }}-\tilde{\mu}$ ( e.g. $\left.\Delta \mu=\mu_{s \downarrow}-\mu_{d \downarrow}\right)$. The Onsager transport equations are modified accordingly. The flux $\overrightarrow{J_{n}}$ and $\dot{\Psi}$ are not of the same tensorial nature (in a first approach): the first is a vector defined on the sphere, and the second is a scalar. According to the Curie principle, Onsager transport coefficients that couple the two processes should not exist. The modification due to the contribution of the electronic relaxation is taken into account by a third relaxation term (we still assume $T$ constant) :

$$
\left\{\begin{array}{l}
J_{n}^{\theta}=-L_{\theta \theta} \frac{\partial \tilde{\mu}}{\partial \theta}-L_{\theta \phi} \frac{1}{\sin \theta} \frac{\partial \tilde{\mu}}{\partial \phi} \\
J_{n}^{\phi}=-L_{\phi \theta} \frac{\partial \tilde{\mu}}{\partial \theta}-L_{\phi \phi} \frac{1}{\sin \theta} \frac{\partial \tilde{\mu}}{\partial \phi} \\
\dot{\Psi}=L \Delta \mu
\end{array}\right.
$$

where $\Delta \mu$ is defined as the chemical affinity of the corresponding reaction, or equivalently, defined as the pumping force associated to the flux $\dot{\Psi}$ of spins transferred between the spinpolarized electric system and the ferromagnet. In other terms, $\Delta \mu$ is the force responsible for irreversible spin-transfer. Integrating over the whole ferromagnetic layer, the Fokker-Planck equation, Eq. (56), rewrites:

$$
\frac{\partial n^{\text {ferro }}}{\partial t}=\frac{\partial n_{0}}{\partial t}+\int_{A}^{B} L_{s d} \Delta \mu(z) d z=0
$$

where the first term in the right hand side $\frac{\partial n_{0}}{\partial t}$ leads to the standard rotational FokkerPlanck equation (defined by the second equation in Eqs. (62) ), and the second term is the 
contribution coming from the electronic relaxation. This equation is the main result that defines the irreversible spin-transfer effect for an open system. Eq. (85) will not be solved here. The aim of the following developments is to define negative damping and effective temperature.

The dynamic equation is obtained in the same way as in the previous section, by writing the flux of representative points on the unit sphere. A term $\frac{d \vec{u}^{e l e c}}{d t}$ (not explicit here) related to the spin-polarized electronic contribution is added to the Eq. (63) and leads to the following generalized LLG equation:

$$
\frac{d \vec{u}}{d t}=\frac{\partial \vec{u}^{\text {elec }}}{\partial t}-g^{\prime} \vec{u} \times\left(\vec{\nabla} V^{m a g}\right)+h^{\prime} \vec{u} \times\left[\vec{u} \times\left(\vec{\nabla} V^{m a g}\right)\right]
$$

Since the effect of the environment conserves the modulus of the magnetization, i.e. $\vec{u} . d \vec{u} / d t=0$ [116], the contribution of $\frac{d \vec{u}}{d t}^{\text {elec }}$ reduces to the two damping factors, parallel to $\vec{u} \times \nabla V^{m a g}$ and parallel to $\vec{u} \times\left(\vec{u} \times \vec{\nabla} V^{m a g}\right)$, and a stochastic force $f(t)$. This necessarily leads to the introduction of two parameters $\alpha_{1}$ and $\alpha_{2}$ such that :

$$
\frac{d \vec{u}}{d t}=-\left(g^{\prime}+\alpha_{1}\right) \vec{u} \times\left(\vec{\nabla} V^{m a g}\right)+\left(h^{\prime}+\alpha_{2}\right) \vec{u} \times\left[\vec{u} \times\left(\vec{\nabla} V^{m a g}\right)\right]+\vec{f}(t)
$$

where the coefficients $\alpha_{1}$ and $\alpha_{2}$ can be thought of as negative damping or positive damping, depending wether the spin transfer $\dot{\Psi}_{s d}$, integrated over the whole layer with the two junctions, is transferred from the electric system to the magnetic system (negative damping) or inversely (positive damping). In other words, it depends on the balance of spin accumulation at the two interfaces of the ferromagnetic layer. Note that as far as the damping coefficients are not explicitly defined, the validity of the argument used above (the Callen's argument [116]) is not restricted to the relaxation and spin accumulation mechanisms described in the first sections of this work, but is much more general. In particular, the equation is formally similar to that described in the framework of the exchange torque or spin torque theory [30, 117, 118, 119, 120, 121, 122]. However, beyond the vectorial argument proposed above, the proper derivation of the reduction from Eq. (86]) to Eq. (87) (see e.g. [41]) is still to be performed. 


\section{Effective thermostat}

The Néel-Brown activation laws describe out-of-equilibrium spin systems $(\partial \tilde{\mu} \neq 0)$, and are valid for high enough potential barriers $k T \ll v V^{m a g}$, or long time scales $\Delta t / \tau_{0} \gg$ 1 , where $\Delta t$ is the measurement time window, and $\tau_{0}$ is the relaxation time scale that describes the coupling to the lattice. If the volume $v$ tends to zero, the energy barrier decreases down to a value such that $v V^{m a g} \leq k T \ln \left(\Delta t / \tau_{0}\right)$, and the magnetization is at "equilibrium" with the lattice for the measurement time window $\Delta t$. The system is no longer metastable but superparamagnetic. The equilibrium imposes the condition $\partial \tilde{\mu}=0$, and the statistical distribution is the Maxwell-Boltzmann distribution $n=N_{t} \exp \left(-v V^{m a g} / k T\right)$. The magnetization behaves like a paramagnetic spin, with the ferromagnetic order parameter $\vec{M}$ instead of the spin $\vec{s}$. In the case of 3 d metallic ferromagnetic nanostructures (Co, $\mathrm{Ni}, \mathrm{Fe} . .$. ) of sizes typically around $10 \mathrm{~nm}$ radius at room temperature, the system is superparamagnetic for time scales of magnetometric measurements (above $10^{-5}$ sec.). The system is nevertheless ferromagnetic and follows the Néel-Brown laws if the measurements are performed in a shorter time window $\Delta t$ (from micro-seconds to nanoseconds in the case of $10 \mathrm{~nm}^{3}$ particles invoked above).

However, if the relevant time window is shorter than the typical ferromagnetic relaxation time scale $\tau_{0}\left(\Delta t \leq \tau_{0}\right)$, the precession now governs the quasi-ballistic dynamics, which is qualitatively different (because it is not driven by the fluctuations). The collective modes measured are that observed with ferromagnetic resonance; e.g. dynamics of thermal spin waves are observed in GMR structures [96, 97, 98, 99, 100]. It is no longer activated, whatever the potential barrier and the volume $v$, and the thermalization process vanishes at short time scales ("quasiballistic magnetization reversal" regime [99]). The temperature of the system (if any [123]) is not necessarily the temperature of the lattice $T_{\text {eff }} \neq T$ : the system is decoupled from the heat bath. A similar situation justified the introduction of the concept of spin temperature $T_{s}$ in the early 50's with the first spin resonance experiments 124]. Note that if the system is also isolated from other sub-systems at comparable time scales, has an upper bound, and if the populations (up and down) can be inverted (like in nuclear spin systems), the spin temperature of the system can even be negative [125, 126] (but the spin temperature is usually positive and higher than the lattice temperature [126]).

In the situation of interest, with spin-polarized currents in $3 \mathrm{~d}$ metallic nanostructures, 
the ferromagnetic order parameter is coupled to the lattice through the polarization of the electronic degrees of freedom [105, 127, 128]. Without being coupled to the magnetization (e.g. in the non- magnetic side of a junction), the spin-accumulation sub-system relaxes toward equilibrium with the relaxation time $\tau_{s f}$ of some picoseconds (as described in the first section with the two channel model). This relaxation time is shorter than the thermalization of the magnetization with the lattice $\tau_{0}$ (nanoseconds). On the other hand, the coupling with the ferromagnetic sub-systems corresponds to a relaxation time $\tau_{s d}$ (comprised between the electronic relaxation time $\tau_{e}$ and $\tau_{s f}$ ) shorter than $\tau_{0}$ : as a consequence, this relaxation "thermalize" the ferromagnetic order parameter with the spin-accumulation sub-system, that takes the role of the heat bath in place of the lattice. This picture is that schematized in Fig [1 in the first section.

In the activation regime, it is possible to assume that the spin-accumulation sub-system is a reservoir of energy, and that the ferromagnetic order parameter is thermalized with it (see Fig. 1). Providing that the spin-accumulation sub-system is not thermalized with the lattice, the zeroth law of thermodynamics is not valid [123], and it is possible to identify it as a thermostat at temperature $T_{\text {eff }}$ in equilibrium with the ferromagnetic system.

The equilibrium condition imposes that $\mathcal{I}=0$ [40]. The entropy production $\mathcal{I}$ of the spin-dependent electric sub-system was calculated in Sec. II. With the temperature $T_{e f f}$ corresponding to the effective equilibrium temperature, the chemical potential writes [38] $\Delta \tilde{\mu}^{e f f}=\Delta \mu^{e f f}+k T_{e f f} \ln \left(n_{\alpha} / n_{\gamma}\right)$.

$$
T_{e f f} \cdot \mathcal{I}=\left(-\frac{\partial \Delta \tilde{\mu}^{e f f}}{\partial z}+2 \epsilon \Delta \tilde{\mu}^{e f f}\right) \delta J+\Delta \tilde{\mu}^{e f f} \dot{\Psi}=0
$$

where the Joule heating contribution $-\frac{\partial \mu_{0}}{\partial z} J_{0}$ has been removed because it does not contribute to the magnetic system and is coupled to the lattice (the whole analysis should also include the Peltier effects: energy can also be extracted from the lattice to the magnetic system).

The entropy production vanishes for the following sufficient condition:

$$
\Delta \tilde{\mu}^{e f f}=0
$$

The condition Eq. (89) leads to the expression of the equilibrium temperature $T_{\text {eff }}$ :

$$
k T_{e f f}=-\frac{\Delta \mu}{\ln \left(n_{\alpha} / n_{\gamma}\right)} \approx-2 \Delta \mu \frac{n_{0}}{\delta n}
$$


where the inversion of population implies that $\delta n \leq 0$. This equation is simply the equilibrium Curie-Weiss law that accounts for the paramagnetic behavior of the spin-accumulation $g \mu_{B} \delta n$ i.e. the first order approximation of the averaging over the Boltzmann distribution at temperature $T_{\text {eff }}$ ( $\delta n$ is the s-d spin accumulation that would be measured with a lattice temperature $T_{\text {eff }}$ ). The evaluation of $\delta n$ would necessitate the non-equilibrium distribution at temperature $\mathrm{T}$ to be calculated. This task is beyond the scope of the present review. However, according to the evaluation performed below, an energy $k T_{\text {eff }} \approx 1 \mathrm{eV}$ can be expected by calculating the ferromagnetic energy under a current of $1 \mathrm{~mA}$ due to the spin transfer in the internal field of $1 T$ of the ferromagnet.

A fundamental consequence of the existence of the effective temperature is that the solution of the stochastic equation of the magnetization is known, and is given by the standard activation equation Eq. (174) with the effective temperature $T_{\text {eff }}$ instead of the lattice temperature $T$ :

$$
\frac{\partial N}{\partial \theta}+\frac{1}{k T_{e f f}} \frac{\partial V}{\partial \theta} N=\frac{I_{e f f}}{2 k_{e f f}^{\prime} \pi \sin (\theta)}
$$

where $I_{\text {eff }}$ is calculated with the Boltzmann distribution with the ferromagnetic energy $\exp \left(V(\theta) / k T_{\text {eff }}\right)$. Assuming an approximatively constant effective temperature $T_{\text {eff }}(\theta) \approx$ $T_{\text {eff }}$, the equation is formally identical to Eq. (74) so that the Néel-Brown activation formula is recovered with $T_{\text {eff }}$ instead of $\mathrm{T}$ :

$$
\left\{\begin{array}{l}
\tau\left(\theta_{1}\right)=\tau_{01} e^{\left(\frac{\Delta V_{1}}{k T_{e f f}}\right)} \\
\tau\left(\theta_{2}\right)=\tau_{02} e^{\left(\frac{\Delta V_{2}}{k T_{e f f}}\right)}
\end{array}\right.
$$

where $\tau_{01}$ and $\tau_{02}$ contain the $k_{\text {eff }}^{\prime}$ dependence. This behavior is experimentally observed 2, 21, 22, 23, 24]. Fig. 10 shows the typical Néel-Brown activation observed with the sample shown in Fig. 8 (b) and (c). The fit of the mean relaxation time as a function of the applied field and the current amplitude injected in the device is performed with the Néel-Brown formula with the effective barrier height as fitting parameter (Fig. 10 (a) and (c)). The effective barrier height as a function of the current is presented in Fig. 10 (c) and (d).

Assuming that the mechanism responsible for $T_{\text {eff }}$ is the spin-accumulation occurring at the interface composed of antialigned ferromagnets, the voltage drop due to spin- 
(a)

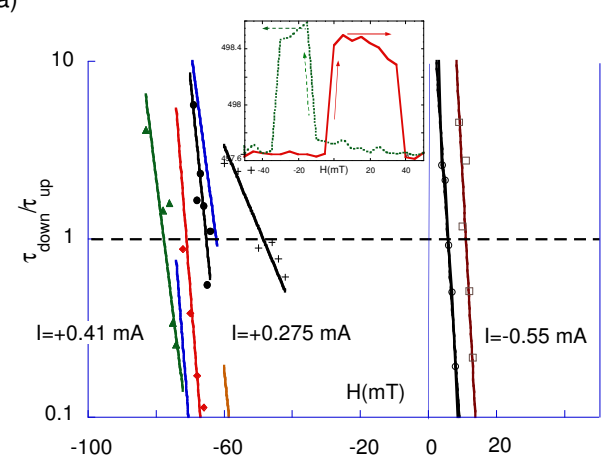

(c)

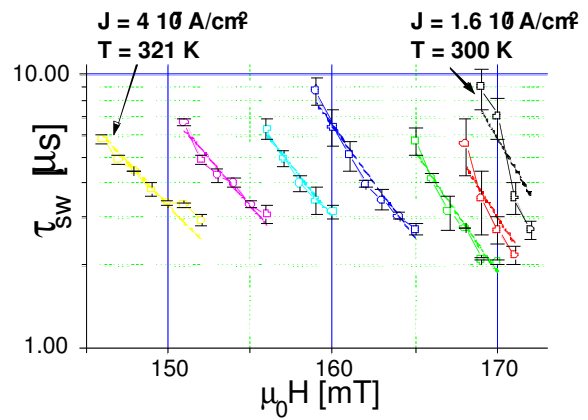

(b)

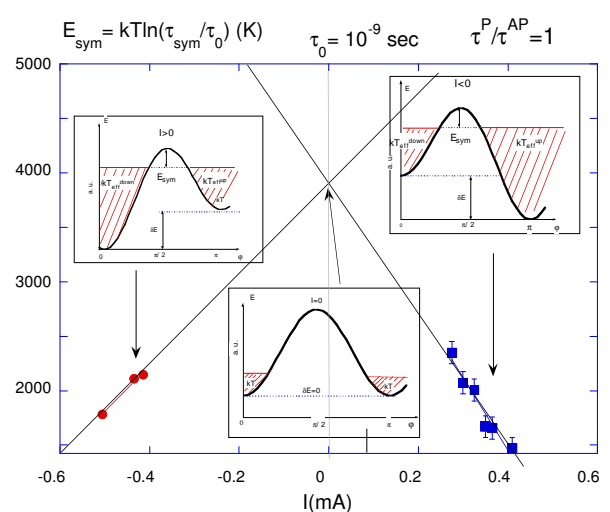

(d)

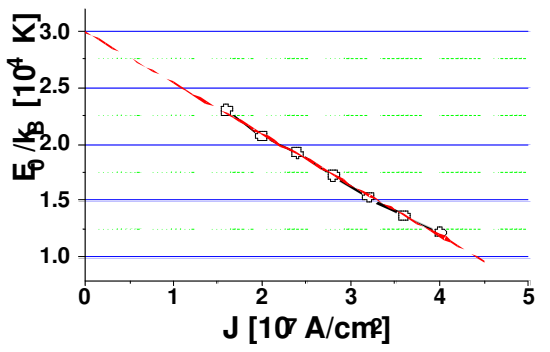

FIG. 10: Observation of the Néel-Brown activation due to current injection in a $\mathrm{Co} / \mathrm{Cu} / \mathrm{Co}$ trilayers (a),(b) and in a Ni nanowire (c),(d). (a) Ratio of the two relaxation times (TLF) and (c) relaxation time as a function of the applied field for different currents fitted with the Néel-Brown formula and effective barrier height as fitting parameter. (b) and (d) Variation of the effective barrier height in Kelvin. In the case of the two level fluctuations, the barrier is measured for the symmetric relaxation times (b) (i.e. for different applied fields). The effect of the fluctuations are sketched in the insets (dashed lines). Reprint with permission from Ref. 22] J.-E. Wegrowe Phys. Rev. B 68, 214414 (2003) and Ph. Guittienne et al., IEEE Trans. Mag-37, 2126 (2001), Copyright@American Physical Society.

accumulation is approximatively equal to $\Delta \mu$ (see Eq. (18)):

$$
k T_{e f f} \approx-\frac{\Delta R^{s a} I}{\ln \left(n_{\alpha} / n_{\gamma}\right)} \propto \frac{\Delta R^{s a} I}{2}
$$

with $-\ln \left(n_{\alpha} / n_{\gamma}\right) \leq 1$ The proportionality between $k T_{e f f}$ and $R^{s a}$ was observed by different groups in recent experimental investigations [130, 131] (with DC measurements, the parameter is the "critical current" $I_{c} \propto T_{\text {eff }}^{-1}$ as shown in Fig. 10(d)).

How to estimate the magnetic energy of the spin-accumulation system in usual experi- 
mental situations, where a current density of some few $\mathrm{mA}$ is injected in the nanostructure 1, 2, 4, 8, 21, 22, 23, 24, 25]? This current corresponds to some $10^{16}$ spin per seconds flowing through the interface. If one assumes that $20 \%$ (polarization of the current) of

the spins are maintained out-of-equilibrium within a typical relaxation time $\tau_{s f}$ of $10^{-11} \sec$, we are left with about $2.10^{4}$ spins that define the magnetization of the spin-accumulation sub-system in the volume defined by the corresponding diffusion length. An effect of the electric spin relaxation on the ferromagnetic order parameter should consequently be expected for a nanostructured ferromagnetic system that is only ten to hundred times larger. In an internal field of $H_{\text {int }}=1 \mathrm{~T}$, this energy $E=10^{4} \mu_{B} H_{\text {int }}$ is of the order $1 \mathrm{eV}$ (beyond the Curie temperature) in accordance with activation experiments performed on various systems 2, 21, 22, 23, 24, 25]. Without current injection, the magnetic order parameter is at room temperature and consequently, the hot sub-system is the spin-accumulation system.

\section{CONCLUSION}

An unified thermokinetics approach of both spin-dependent charge carriers and ferromagnetic Brownian motion has been presented in the context of open systems. The spindependent electronic relaxation is then introduced as a source term in the conservation equation of the magnetic moment. This leads to the description of the effect of spin-injection induced magnetization switching, or irreversible spin-transfer in an open ferromagnetic layer.

The description of the spin-accumulated charge carriers is based on the two conduction channel approximation, generalized to both intra -and inter- band relaxation. The application of the first and second laws of thermodynamics, together with the conservation laws, lead to the spin-dependent transport equations. The relevant Onsager transport coefficients are introduced and related to the typical electronic relaxation times. The effect of charge conservation and screening is also taken into account.

On the other hand, the ferromagnetic order parameter is described on an equal footing by introducing the conservation laws and the relevant chemical potential, with deterministic terms accounting for the effective field, and dissipative terms accounting for the coupling to a relevant heat bath. The corresponding Onsager transport coefficients are defined with the second law of thermodynamics, and refined with the help of the Onsager reciprocal relations. The rotational Fokker-Planck equation, and the Landau-Lifshitz-Gilbert (LLG) 
equation are then derived within the thermokinetic theory. The Onsager coefficients are related to the typical time scales of the ferromagnetic relaxation $\left(\tau_{0}\right)$. In the activation regime, the Néel-Brown activation law is deduced.

In the framework of this description, the generalization of both the Fokker-Planck equation and the LLG equation with adding the contribution of spin-accumulation is straightforward in terms of flux of representative points in the magnetization sphere. The negative damping appears naturally in order to describe the exchange of spins from the electric subsystem to the magnetic sub-system, described as a coupling to an environment.

Furthermore, the discussion about the different relaxation times shows that the spinpolarized current is not thermalized to the lattice in the stationary regime, but is thermalized with the spin-accumulation sub-system. The argument is that on one hand the relaxation toward equilibrium of the spin-accumulation system (described by $\tau_{s f}$ : some tens to hundreds

of picoseconds) is shorter than the thermalization of the ferromagnetic system (described by $\tau_{0}$ : nanoseconds). And on the other hand, the coupling between the ferromagnetic order parameter and the spin-accumulation sub-system $\left(\tau_{s d}\right)$ is shorter or equal to the $\tau_{s f}$.

An effective temperature is then derived in the activation regime through the entropy production, and leads to the derivation of an effective Néel-Brown relaxation process due to current injection, that is experimentally observed.

\section{ACKNOWLEDGEMENT}

We are indebted to Prof. Peter Levy for the constant interest along this work and for a critical reading of the manuscript. 


\section{APPENDIX A: MICROSCOPIC APPROACH AND THERMOKINETIC COEF-}

\section{FICIENTS}

\section{Relation between $L$ and the electronic relaxation times}

Let us consider a simple interface between two metals. Far from the interface, the Ohm's law is recovered : the chemical potentials of the channels are identical and the electric distribution is that of equilibrium $n_{\alpha 0}$ and $n_{\gamma 0}$. In the following, we assume that the charge transfer between the two channels can be described by the following relation:

$$
f \Delta n_{\alpha}(x)+g \Delta n_{\gamma}(x)=0
$$

The case $f=g=1$ describes the local electrical neutrality. We have:

$$
\left\{\begin{array}{l}
\Delta n_{\alpha}(x)=\left(\mu_{c h, \alpha}-\mu^{0}\right) N_{\alpha}\left(E_{F}\right) \\
\Delta n_{\gamma}(x)=\left(\mu_{c h, \gamma}-\mu^{0}\right) N_{\gamma}\left(E_{F}\right)
\end{array}\right.
$$

where $\mu^{0}$ is the chemical potential in the absence of charge transfer and $\mu_{c h, \alpha}$ and $\mu_{c h, \gamma}$ are the purely chemical potentials of the channels (without transfer $\mu_{c h, \alpha}=\mu_{c h, \gamma}=\mu_{0}$ ) ; $N_{\alpha, \gamma}\left(E_{F}\right)$ is the density of states at the Fermi level. The separation between the electric potential and the purely chemical potential writes:

$$
\left\{\begin{array}{l}
\mu_{\alpha}=\mu_{c h, \alpha}+e V \\
\mu_{\gamma}=\mu_{c h, \gamma}+e V
\end{array}\right.
$$

where $\mathrm{V}$ is the local electric potential.

Relation (A92) gives

$$
\left(\mu_{c h, \alpha}-\mu^{0}\right) f N_{\alpha}\left(E_{F}\right)+\left(\mu_{c h, \gamma}-\mu^{0}\right) g N_{\gamma}\left(E_{F}\right)=0
$$

Note that the expressions of $\Delta n_{\alpha, \gamma}$ comes from the fact that at zero Kelvin:

$$
\left\{\begin{array}{l}
n_{\alpha}\left(\mu_{\alpha}\right)=\int N_{\alpha}(E) f(E) d E=\int_{-\infty}^{\mu_{c h, \alpha}} N_{\alpha}(E) f(E) d E \\
n_{\gamma}\left(\mu_{\gamma}\right)=\int N_{\gamma}(E) f(E) d E=\int_{-\infty}^{\mu_{c h, \gamma}} N_{\gamma}(E) f(E) d E
\end{array}\right.
$$


From these relations we deduce:

$$
\left\{\begin{array} { l } 
{ \Delta n _ { \alpha } = N _ { \alpha } ( E _ { F } ) \delta \mu _ { c h , \alpha } } \\
{ \Delta n _ { \gamma } = N _ { \gamma } ( E _ { F } ) \delta \mu _ { c h , \gamma } = - \frac { f } { g } \Delta n _ { \alpha } }
\end{array} \Rightarrow \left\{\begin{array}{l}
\Delta n_{\alpha}=\frac{g N_{\alpha}\left(E_{F}\right) N_{\gamma}\left(E_{F}\right)}{f N_{\alpha}\left(E_{F}\right)+g N_{\gamma}\left(E_{F}\right)} \delta \mu_{c h, \alpha} \\
\Delta n_{\gamma}=-\frac{f N_{\alpha}\left(E_{F}\right) N_{\gamma}\left(E_{F}\right)}{f N_{\alpha}\left(E_{F}\right)+g N_{\gamma}\left(E_{F}\right)} \delta \mu_{c h, \alpha}
\end{array}\right.\right.
$$

where $\delta \mu_{\alpha \gamma}=\mu_{c h, \alpha \gamma}-\mu_{0}$.

Introducing the global transfer rate $T_{\alpha \rightarrow \gamma}\left(\right.$ resp. $\left.T_{\gamma \rightarrow \alpha}\right)$ of the channel $\alpha$ to $\gamma$ (resp. $\gamma$ to $\alpha$ ), the charge conservation between the two channels writes $(e<0)$ :

$$
\left\{\begin{array}{l}
\frac{\partial n_{\alpha}(x)}{\partial t}=-\frac{1}{e} \frac{\partial J_{\alpha}(x)}{\partial x}-T_{\alpha \rightarrow \gamma}\left(n_{\alpha}(x), n_{\gamma}(x)\right)+T_{\gamma \rightarrow \alpha}\left(n_{\alpha}(x), n_{\gamma}(x)\right) \\
\frac{\partial n_{\gamma}(x)}{\partial t}=-\frac{1}{e} \frac{\partial J_{\gamma}(x)}{\partial x}+T_{\alpha \rightarrow \gamma}\left(n_{\alpha}(x), n_{\gamma}(x)\right)-T_{\gamma \rightarrow \alpha}\left(n_{\alpha}(x), n_{\gamma}(x)\right)
\end{array}\right.
$$

that leads, in the stationary regime $\frac{\partial n_{\alpha, \gamma}(x)}{\partial t}=0$, to the following relations:

$$
\left\{\begin{array}{l}
\frac{\partial J_{\alpha}(x)}{\partial x}=-e T_{\alpha \rightarrow \gamma}\left(n_{\alpha}(x), n_{\gamma}(x)\right)+e T_{\gamma \rightarrow \alpha}\left(n_{\alpha}(x), n_{\gamma}(x)\right) \\
\frac{\partial J_{\gamma}(x)}{\partial x}=+e T_{\alpha \rightarrow \gamma}\left(n_{\alpha}(x), n_{\gamma}(x)\right)-e T_{\gamma \rightarrow \alpha}\left(n_{\alpha}(x), n_{\gamma}(x)\right)
\end{array}\right.
$$

The Taylor expansion to the leading order of the transfer rates $T_{\alpha \rightarrow \gamma}$ and $T_{\gamma \rightarrow \alpha}$, around equilibrium gives:

$$
\left\{\begin{aligned}
\frac{\partial J_{\alpha}(x)}{\partial x}=- & e T_{\alpha \rightarrow \gamma}\left(n_{\alpha}^{0}, n_{\gamma}^{0}\right)-e \frac{\partial T_{\alpha \rightarrow \gamma}}{\partial n_{\alpha}} \Delta n_{\alpha}-e \frac{\partial T_{\alpha \rightarrow \gamma}}{\partial n_{\gamma}} \Delta n_{\gamma} \\
& +e T_{\gamma \rightarrow \alpha}\left(n_{\alpha}^{0}, n_{\gamma}^{0}\right)+e \frac{\partial T_{\gamma \rightarrow \alpha}}{\partial n_{\alpha}} \Delta n_{\alpha}+e \frac{\partial T_{\gamma \rightarrow \alpha}}{\partial n_{\gamma}} \Delta n_{\gamma} \\
\frac{\partial J_{\gamma}(x)}{\partial x}=- & \frac{\partial J_{\alpha}(x)}{\partial x}
\end{aligned}\right.
$$

At equilibrium, the current of each channel is conserved, so that:

$$
-e T_{\alpha \rightarrow \gamma}\left(n_{\alpha}^{0}, n_{\gamma}^{0}\right)+e T_{\gamma \rightarrow \alpha}\left(n_{\alpha}^{0}, n_{\gamma}^{0}\right)=0
$$

Defining the electronic relaxation times:

$\tau_{\alpha \rightarrow \gamma}, \tau_{\gamma \rightarrow \alpha}$, such that

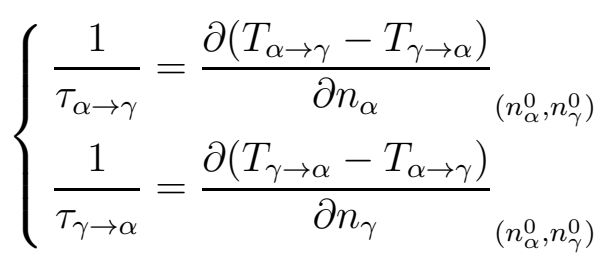


we have :

$$
\begin{gathered}
\left\{\begin{array}{l}
\frac{\partial J_{\alpha}(x)}{\partial x}=-e \frac{\Delta n_{\alpha}}{\tau_{\alpha \rightarrow \gamma}}+e \frac{\Delta n_{\gamma}}{\tau_{\gamma \rightarrow \alpha}} \\
\frac{\partial J_{\gamma}(x)}{\partial x}=-\frac{\partial J_{\alpha}(x)}{\partial x}
\end{array} \Rightarrow\right. \\
\left\{\begin{array}{l}
\frac{\partial J_{\alpha}(x)}{\partial x}=-e \frac{N_{\alpha}\left(E_{F}\right) N_{\gamma}\left(E_{F}\right)}{f N_{\alpha}\left(E_{F}\right)+g N_{\gamma}\left(E_{F}\right)}\left(\frac{g}{\tau_{\alpha \rightarrow \gamma}}+\frac{f}{\tau_{\gamma \rightarrow \alpha}}\right)\left(\mu_{c h, \alpha}-\mu_{c h, \gamma}\right) \\
\frac{\partial J_{\gamma}(x)}{\partial x}=-\frac{\partial J_{\alpha}(x)}{\partial x}
\end{array}\right.
\end{gathered}
$$

The above equations can be rewritten in the following form :

$$
\left\{\begin{array}{l}
\frac{\partial J_{\alpha}(x)}{\partial x}=-L\left(\mu_{c h, \alpha}-\mu_{c h, \gamma}\right)=-L\left(\mu_{\alpha}-\mu_{\gamma}\right) \\
\frac{\partial J_{\gamma}(x)}{\partial x}=+L\left(\mu_{c h, \alpha}-\mu_{c h, \gamma}\right)=+L\left(\mu_{\alpha}-\mu_{\gamma}\right)
\end{array}\right.
$$

where the coefficient Onsager transport coefficient $L$ is related to the electronic relaxation times by the following relation:

$$
L=e \frac{N_{\alpha}\left(E_{F}\right) N_{\gamma}\left(E_{F}\right)}{f N_{\alpha}\left(E_{F}\right)+g N_{\gamma}\left(E_{F}\right)}\left(\frac{g}{\tau_{\alpha \rightarrow \gamma}}+\frac{f}{\tau_{\gamma \rightarrow \alpha}}\right)
$$

The form Eq. (A105) is that of Eq. (9) deduced from the thermokinetic approach in Sec. I, where the coefficient $L$ is the Onsager coefficient defined in the third equation of Eqs. (11).

\section{Determination of $f$ and $g$}

We have, for the stationary regime:

$$
\frac{\partial\left(J_{\alpha}(x)-J_{\gamma}(x)\right)}{\partial x}=-2 L\left(\mu_{\alpha}-\mu_{\gamma}\right)
$$

Furthermore, the local Ohm's law applied to each channel leads to the following equations:

$$
\left\{\begin{array} { l } 
{ J _ { \alpha } = - \frac { \sigma _ { \alpha } } { e } \frac { \partial \mu _ { \alpha } ( x ) } { \partial x } } \\
{ J _ { \gamma } = - \frac { \sigma _ { \gamma } } { e } \frac { \partial \mu _ { \gamma } ( x ) } { \partial x } }
\end{array} \Rightarrow \left\{\begin{array}{l}
\frac{\partial J_{\alpha}(x)}{\partial x}=-\frac{\sigma_{\alpha}}{e} \frac{\partial^{2} \mu_{\alpha}(x)}{\partial x^{2}} \\
\frac{\partial J_{\gamma}(x)}{\partial x}=-\frac{\sigma_{\gamma}}{e} \frac{\partial^{2} \mu_{\gamma}(x)}{\partial x^{2}}
\end{array}\right.\right.
$$


where we assume that the conductivities are constant.

From (A105) and (A107), we get:

$$
\left\{\begin{array}{l}
\frac{\partial^{2} \mu_{\alpha}(x)}{\partial x^{2}}=\frac{e L}{\sigma_{\alpha}}\left(\mu_{\alpha}-\mu_{\gamma}\right) \\
\frac{\partial^{2} \mu_{\gamma}(x)}{\partial x^{2}}=-\frac{e L}{\sigma_{\gamma}}\left(\mu_{\alpha}-\mu_{\gamma}\right)
\end{array} \Rightarrow \frac{\partial^{2}\left(\mu_{\alpha}-\mu_{\gamma}\right)}{\partial x^{2}}=e L\left(\frac{1}{\sigma_{\alpha}}+\frac{1}{\sigma_{\gamma}}\right)\left(\mu_{\alpha}-\mu_{\gamma}\right)\right.
$$

that leads to the well-known diffusion equation of the chemical potential, that describes the spin-accumulation process:

$$
\frac{\partial^{2} \Delta \mu}{\partial x^{2}}=e L\left(\frac{1}{\sigma_{\alpha}}+\frac{1}{\sigma_{\gamma}}\right) \Delta \mu
$$

from which the spin-diffusion length $l_{s f}$ is deduced:

$$
\frac{1}{l_{s f}^{2}}=e L\left(\frac{1}{\sigma_{\alpha}}+\frac{1}{\sigma_{\gamma}}\right)
$$

From Eq. (A108) we have the differential equations:

$$
\left\{\begin{array}{l}
\frac{\partial^{2} \mu_{\alpha}(x)}{\partial x^{2}}=\frac{e L}{\sigma_{\alpha}} \Delta \mu=\frac{\sigma_{\gamma}}{\sigma_{t}} \frac{\partial^{2} \Delta \mu}{\partial x^{2}} \\
\frac{\partial^{2} \mu_{\gamma}(x)}{\partial x^{2}}=-\frac{e L}{\sigma_{\gamma}} \Delta \mu=-\frac{\sigma_{\alpha}}{\sigma_{t}} \frac{\partial^{2} \Delta \mu}{\partial x^{2}}
\end{array}\right.
$$

\section{Charge distribution and screening}

Separating the electric contribution from the chemical contribution, the electrochemical potential writes :

$$
\mu_{\alpha, \gamma}=\mu_{c h, \alpha, \gamma}+e V
$$

so that:

$$
\left\{\begin{array} { l } 
{ \frac { \partial ^ { 2 } \mu _ { \alpha } ( x ) } { \partial x ^ { 2 } } = \frac { \partial ^ { 2 } \mu _ { c h , \alpha } ( x ) } { \partial x ^ { 2 } } + e \frac { \partial ^ { 2 } V ( x ) } { \partial x ^ { 2 } } } \\
{ \frac { \partial ^ { 2 } \mu _ { \gamma } ( x ) } { \partial x ^ { 2 } } = \frac { \partial ^ { 2 } \mu _ { c h , \gamma } ( x ) } { \partial x ^ { 2 } } + e \frac { \partial ^ { 2 } V ( x ) } { \partial x ^ { 2 } } }
\end{array} \Rightarrow \left\{\begin{array}{l}
\frac{\partial^{2} \mu_{c h, \alpha}(x)}{\partial x^{2}}=\frac{\sigma_{\gamma}}{\sigma_{t}} \frac{\partial^{2} \Delta \mu}{\partial x^{2}}+e^{2} \frac{\Delta n_{\alpha}+\Delta n_{\gamma}}{\epsilon} \\
\frac{\partial^{2} \mu_{c h, \gamma}(x)}{\partial x^{2}}=-\frac{\sigma_{\alpha}}{\sigma_{t}} \frac{\partial^{2} \Delta \mu}{\partial x^{2}}+e^{2} \frac{\Delta n_{\alpha}+\Delta n_{\gamma}}{\epsilon}
\end{array}\right.\right.
$$

where the Poisson equation has been introduced

$$
-\frac{\partial^{2} V(x)}{\partial x^{2}}=e \frac{\Delta n_{\alpha}+\Delta n_{\gamma}}{\epsilon}
$$


The equations rewrite:

$$
\left\{\begin{array}{l}
\frac{\partial^{2} \delta \mu_{c h, \alpha}(x)}{\partial x^{2}}-e^{2} \frac{N_{\alpha} \delta \mu_{c h, \alpha}+N_{\gamma} \delta \mu_{c h, \gamma}}{\epsilon}=\frac{\sigma_{\gamma}}{\sigma_{t}} \frac{\Delta \mu}{l_{s f}^{2}} \\
\frac{\partial^{2} \delta \mu_{c h, \gamma}(x)}{\partial x^{2}}-e^{2} \frac{N_{\alpha} \delta \mu_{c h, \alpha}+N_{\gamma} \delta \mu_{c h, \gamma}}{\epsilon}=-\frac{\sigma_{\alpha}}{\sigma_{t}} \frac{\Delta \mu}{l_{s f}^{2}}
\end{array}\right.
$$

These relations with

$$
\mu_{\alpha}-\mu_{\gamma}=\mu_{c h, \alpha}-\mu_{c h, \gamma}=\delta \mu_{c h, \alpha}-\delta \mu_{c h, \gamma}
$$

lead to

$$
\left\{\begin{array}{l}
\frac{\partial^{2} \Delta \mu_{c h, \alpha}(x)}{\partial x^{2}}-\frac{\Delta \mu_{c h, \alpha}}{l^{2}}=\Delta \mu\left(\frac{\sigma_{\gamma}}{\sigma_{t} l_{s f}^{2}}-e^{2} \frac{N_{\gamma}}{\epsilon}\right) \\
\frac{\partial^{2} \Delta \mu_{c h, \gamma}(x)}{\partial x^{2}}-\frac{\Delta \mu_{c h, \gamma}}{l^{2}}=\Delta \mu\left(-\frac{\sigma_{\alpha}}{\sigma_{t} l_{s f}^{2}}+e^{2} \frac{N_{\alpha}}{\epsilon}\right)
\end{array}\right.
$$

where we have introduced the screening length :

$$
\frac{1}{l^{2}}=e^{2} \frac{N_{\alpha}+N_{\gamma}}{\epsilon}
$$

The solution of the equation is composed by a solution of the equation with zero right hand side (homogeneous solution) and a particular solution.

1. Solution for $\Delta \mu_{c h, \alpha}$

Homogeneous solution

$$
\Delta \mu_{c h, \alpha}^{h m g}=A \exp \left(\frac{x}{l}\right)+B \exp \left(-\frac{x}{l}\right)
$$

Particular solution $\Delta \mu_{c h, \alpha}^{\text {part }}=\mathrm{p}_{\alpha} \Delta \mu$

$$
\begin{gathered}
\Rightarrow \mathrm{p}_{\alpha} \frac{\Delta \mu}{l_{s f}^{2}}-\frac{\Delta \mu}{l^{2}}=\left(\frac{\sigma_{\gamma}}{\sigma_{t} l_{s f}^{2}}-e^{2} \frac{N_{\gamma}}{\epsilon}\right) \Delta \mu \\
\Rightarrow \mathrm{p}_{\alpha}=\frac{\frac{\sigma_{\gamma}}{\sigma_{t} l_{s f}^{2}}-\frac{e^{2} N_{\gamma}}{\epsilon}}{\frac{1}{l_{s f}^{2}}-\frac{1}{l^{2}}}
\end{gathered}
$$


2. Solution for $\Delta \mu_{c h, \gamma}$

Homogeneous solution

$$
\Delta \mu_{c h, \gamma}^{h m g}=A^{\prime} \exp \left(\frac{x}{l}\right)+B^{\prime} \exp \left(-\frac{x}{l}\right)
$$

Particular solution: $\Delta \mu_{c h, \gamma}^{\text {part }}=\mathrm{p}_{\gamma} \Delta \mu$

$$
\begin{gathered}
\Rightarrow \mathrm{p}_{\gamma} \frac{\Delta \mu}{l_{s f}^{2}}-\frac{\Delta \mu}{l^{2}}=\left(\frac{-\sigma_{\alpha}}{\sigma_{t} l_{s f}^{2}}+e^{2} \frac{N_{\alpha}}{\epsilon}\right) \Delta \mu \\
\Rightarrow \mathrm{p}_{\gamma}=\frac{-\frac{\sigma_{\alpha}}{\sigma_{t} l_{s f}^{2}}+\frac{e^{2} N_{\alpha}}{\epsilon}}{\frac{1}{l_{s f}^{2}}-\frac{1}{l^{2}}}
\end{gathered}
$$

The general solutions satisfying the condition

$$
\Delta \mu_{c h, \alpha}-\Delta \mu_{c h, \gamma}=\Delta \mu
$$

correspond to

$$
A=A^{\prime} \quad B=B^{\prime}
$$

Inserted in the expression of the charge conservation

$$
f \Delta n_{\alpha}+g \Delta n_{\gamma}=0
$$

we have

$$
\begin{gathered}
f N_{\alpha}\left(A \exp \left(\frac{x}{l}\right)+B \exp \left(-\frac{x}{l}\right)+\mathrm{p}_{\alpha} \Delta \mu\right)+g N_{\gamma}\left(A \exp \left(\frac{x}{l}\right)+B \exp \left(-\frac{x}{l}\right)+\mathrm{p}_{\gamma} \Delta \mu\right)=0 \\
\Rightarrow\left(f N_{\alpha}+g N_{\gamma}\right)\left(A \exp \left(\frac{x}{l}\right)+B \exp \left(-\frac{x}{l}\right)\right)+\left(f N_{\alpha} \mathrm{p}_{\alpha}+g N_{\gamma} \mathrm{p}_{\gamma}\right) \Delta \mu=0, \text { for all } x \Rightarrow \\
f N_{\alpha} \mathrm{p}_{\alpha}+g N_{\gamma} \mathrm{p}_{\gamma}=0 \quad \text { and } \quad A=B=0 \\
\Rightarrow f N_{\alpha}\left(\frac{\sigma_{\gamma}}{\sigma_{t} l_{s f}^{2}}-\frac{e^{2} N_{\gamma}}{\epsilon}\right)+g N_{\gamma}\left(-\frac{\sigma_{\alpha}}{\sigma_{t} l_{s f}^{2}}+\frac{e^{2} N_{\alpha}}{\epsilon}\right)=0
\end{gathered}
$$

A solution writes: 


$$
\left\{\begin{array}{l}
f=N_{\gamma}\left(\frac{\sigma_{\alpha}}{\sigma_{t} l_{s f}^{2}}-\frac{e^{2} N_{\alpha}}{\epsilon}\right) \\
g=N_{\alpha}\left(\frac{\sigma_{\gamma}}{\sigma_{t} l_{s f}^{2}}-\frac{e^{2} N_{\gamma}}{\epsilon}\right)
\end{array}\right.
$$

\section{4. $l_{s f}$ function of $l_{\alpha}, l_{\gamma}$ and $l$}

The relation between $L$ and the electronic relaxation times has been found to be:

$$
L=e \frac{N_{\alpha}\left(E_{F}\right) N_{\gamma}\left(E_{F}\right)}{f N_{\alpha}\left(E_{F}\right)+g N_{\gamma}\left(E_{F}\right)}\left(\frac{g}{\tau_{\alpha \rightarrow \gamma}}+\frac{f}{\tau_{\gamma \rightarrow \alpha}}\right)
$$

Inserting the expression of $f$ and $g$ obtained in the previous paragraph, $L$ becomes:

$$
L=e \frac{\frac{1}{\sigma_{t} l_{s f}^{2}}\left(\frac{\sigma_{\alpha} N_{\gamma}}{\tau_{\gamma \rightarrow \alpha}}+\frac{\sigma_{\gamma} N_{\alpha}}{\tau_{\alpha \rightarrow \gamma}}\right)-e^{2} \frac{N_{\alpha} N_{\gamma}}{\epsilon}\left(\frac{1}{\tau_{\alpha \rightarrow \gamma}}+\frac{1}{\tau_{\gamma \rightarrow \alpha}}\right)}{\frac{1}{l_{s f}^{2}}-\frac{1}{l^{2}}}
$$

Furthermore, according to Eq. (A110), the coefficient $L$ can also be written in the following form:

$$
L=\frac{\sigma_{\alpha} \sigma_{\gamma}}{e \sigma_{t} l_{s f}^{2}}
$$

Both results lead to the equation:

$$
e^{2} \frac{\frac{1}{\sigma_{t} l_{s f}^{2}}\left(\frac{\sigma_{\alpha} N_{\gamma}}{\tau_{\gamma \rightarrow \alpha}}+\frac{\sigma_{\gamma} N_{\alpha}}{\tau_{\alpha \rightarrow \gamma}}\right)-e^{2} \frac{N_{\alpha} N_{\gamma}}{\epsilon}\left(\frac{1}{\tau_{\alpha \rightarrow \gamma}}+\frac{1}{\tau_{\gamma \rightarrow \alpha}}\right)}{\frac{1}{l_{s f}^{2}}-\frac{1}{l^{2}}}=\frac{\sigma_{\alpha} \sigma_{\gamma}}{\sigma_{t} l_{s f}^{2}}
$$

Let us define the typical diffusion length per channel $l_{\alpha}$ et $l_{\gamma}$ such that

$$
\left\{\begin{array} { l } 
{ l _ { \alpha } ^ { 2 } = \frac { \sigma _ { \alpha } } { e ^ { 2 } N _ { \alpha } } \tau _ { \alpha \rightarrow \gamma } } \\
{ l _ { \gamma } ^ { 2 } = \frac { \sigma _ { \gamma } } { e ^ { 2 } N _ { \gamma } } \tau _ { \gamma \rightarrow \alpha } }
\end{array} \Rightarrow \left\{\begin{array} { l } 
{ \frac { 1 } { \tau _ { \alpha \rightarrow \gamma } } = \frac { \sigma _ { \alpha } } { e ^ { 2 } N _ { \alpha } l _ { \alpha } ^ { 2 } } } \\
{ \frac { 1 } { \tau _ { \gamma \rightarrow \alpha } } = \frac { \sigma _ { \gamma } } { e ^ { 2 } N _ { \gamma } l _ { \gamma } ^ { 2 } } }
\end{array} \Rightarrow \left\{\begin{array}{l}
\frac{\sigma_{\alpha} N_{\gamma}}{\tau_{\gamma \rightarrow \alpha}}=\frac{\sigma_{\gamma} \sigma_{\alpha}}{e^{2} l_{\gamma}^{2}} \\
\frac{\sigma_{\gamma} N_{\alpha}}{\tau_{\alpha \rightarrow \gamma}}=\frac{\sigma_{\gamma} \sigma_{\alpha}}{e^{2} l_{\alpha}^{2}}
\end{array}\right.\right.\right.
$$

Eq. (A136) rewrites:

$$
\begin{gathered}
\frac{\frac{\sigma_{\alpha} \sigma_{\gamma}}{e^{2} \sigma_{t} l_{s f}^{2}}\left(\frac{1}{l_{\gamma}^{2}}+\frac{1}{l_{\alpha}^{2}}\right)-\frac{N_{\alpha} N_{\gamma}}{\epsilon}\left(\frac{\sigma_{\alpha}}{N_{\alpha} l_{\alpha}^{2}}+\frac{\sigma_{\gamma}}{N_{\gamma} l_{\gamma}^{2}}\right)}{\frac{1}{l_{s f}^{2}}-\frac{1}{l^{2}}}=\frac{\sigma_{\alpha} \sigma_{\gamma}}{e^{2} \sigma_{t} l_{s f}^{2}} \Rightarrow \\
\frac{\sigma_{t}}{N_{\alpha}+N_{\gamma}}\left(\frac{N_{\gamma}}{\sigma_{\gamma} l_{\alpha}^{2}}+\frac{N_{\alpha}}{\sigma_{\alpha} l_{\gamma}^{2}}\right) l_{s f}^{4}-\left[l^{2}\left(\frac{1}{l_{\gamma}^{2}}+\frac{1}{l_{\alpha}^{2}}\right)+1\right] l_{s f}^{2}+l^{2}=0
\end{gathered}
$$


a. Limits: In metals, the screening length is much smaller than the diffusion length of both channels:

1. $l / l_{\alpha \gamma} \ll 0$

$$
\frac{\sigma_{t}}{N_{\alpha}+N_{\gamma}}\left(\frac{N_{\gamma}}{\sigma_{\gamma} l_{\alpha}^{2}}+\frac{N_{\alpha}}{\sigma_{\alpha} l_{\gamma}^{2}}\right) l_{s f}^{2}-1=0 \Rightarrow \frac{1}{l_{s f}^{2}}=\frac{N_{\gamma} /\left(N_{\alpha}+N_{\gamma}\right)}{\sigma_{\gamma} /\left(\sigma_{\alpha}+\sigma_{\gamma}\right)} \frac{1}{l_{\alpha}^{2}}+\frac{N_{\alpha} /\left(N_{\alpha}+N_{\gamma}\right)}{\sigma_{\alpha} /\left(\sigma_{\alpha}+\sigma_{\gamma}\right)} \frac{1}{l_{\gamma}^{2}}
$$

The other limit gives:

2. $l / l_{\alpha \gamma} \Rightarrow \infty$

$$
l^{2}\left[1-\left(\frac{1}{l_{\alpha}^{2}}+\frac{1}{l_{\gamma}^{2}}\right) l_{s f}^{2}\right]=0 \Rightarrow \frac{1}{l_{s f}^{2}}=\frac{1}{l_{\alpha}^{2}}+\frac{1}{l_{\gamma}^{2}}
$$

But this second limit is not expected in usual materials.

\section{APPENDIX B: ONSAGER MATRIX}

The aim of this Appendix is to derive the Onsager matrix (28), (and as a particular case (11) ) on the basis of the first and second laws of thermodynamics. In a typical one dimensional junction the layer is decomposed into $\Omega$ parts, defining the sub-system $\Sigma^{k}$, which is in contact to the "reservoirs" $\Sigma^{k-1}$ and $\Sigma^{k+1}$. The sub-systems $\Sigma^{k}$, is then an open system which exchanges heat and chemical species with its left and right vicinity layers.

Furthermore, the populations $\left(N_{s \uparrow}^{k}, N_{s \downarrow}^{k}, N_{d \uparrow}^{k}\right)$ and spin down $\left(N_{d \downarrow}^{k}\right)$ are not conserved due to transitions from one channel to the other.

In this picture, the states of the sub-system $\Sigma^{k}$ are described by the variables

$$
\left(S^{k}, N_{s \uparrow}^{k}, N_{s \downarrow}^{k}, N_{d \uparrow}^{k}, N_{d \downarrow}^{k}\right)
$$

where $S^{k}$ is the entropy. The internal variables $\Psi_{s}, \Psi_{d}$ and $\Psi_{s d}$ must however be introduced in order to take into account the relaxation processes occurring respectively between the two s-spin channels, the two d-spin channels, and the s-d relaxation .

Let us define the heat and chemical power by $P_{\phi}$ (the mechanical power is zero as long as the action of the magnetic field on the charge carriers is neglected). The first law of the thermodynamics applied to the layer $\Sigma^{k}$ gives

$$
\frac{d E^{k}}{d t}=P_{\phi}^{k-1 \rightarrow k}-P_{\phi}^{k \rightarrow k+1}
$$


Introducing the canonical definitions $T^{k}=\frac{\partial E^{k}}{\partial S^{k}}$ and $\mu_{s \pm}^{k}=\frac{\partial E^{k}}{\partial N_{s \pm}^{k}}, \mu_{d \pm}^{k}=\frac{\partial E^{k}}{\partial N_{d \pm}^{k}}$ the energy variation is:

$$
\frac{d E^{k}}{d t}=T^{k} \frac{d S^{k}}{d t}+\mu_{s \uparrow}^{k} \frac{d N_{s \uparrow}^{k}}{d t}+\mu_{s \downarrow}^{k} \frac{d N_{s \downarrow}^{k}}{d t}+\mu_{d \uparrow}^{k} \frac{d N_{d \uparrow}^{k}}{d t}+\mu_{d \downarrow}^{k} \frac{d N_{d \downarrow}^{k}}{d t}
$$

For the sake of simplicity, we limit our analysis to the isothermal case, $T^{k}=T$. The entropy variation of the sub-layer is deduced from the two last equations, after introducing the conservation laws and after defining the polarized currents $\delta I_{\downarrow}=I_{s \downarrow}-I_{d \downarrow}, \delta I_{\downarrow}=$ $I_{s \downarrow}-I_{d \downarrow}$, and the currents $I_{\downarrow}=I_{s \downarrow}+I_{d \downarrow}, I_{s}=I_{s \uparrow}+I_{s \downarrow}$,

$$
\begin{aligned}
T \frac{d S^{k}}{d t}= & P_{\phi}^{k-1 \rightarrow k}-P_{\phi}^{k \rightarrow k+1}-\frac{1}{2} \Delta \mu_{s}^{k}\left(\delta I_{\downarrow}^{k-1 \rightarrow k}-\delta I_{\downarrow}^{k \rightarrow k+1}+\dot{\Psi}_{s d}^{k}-2 \dot{\Psi}_{s}^{k}\right) \\
& -\frac{1}{2} \mu_{s}^{k}\left(I_{s}^{k-1 \rightarrow k}-I_{s}^{k \rightarrow k+1}-\dot{\Psi}_{s d}^{k}\right)-\frac{1}{2} \Delta \mu_{\downarrow}^{k}\left(\delta I_{\downarrow}^{k-1 \rightarrow k}-\delta I_{\downarrow}^{k \rightarrow k+1}-2 \dot{\Psi}_{s d}^{k}-\dot{\Psi}_{s}^{k}\right) \\
& -\frac{1}{2} \mu_{\downarrow}^{k}\left(I_{\downarrow}^{k-1 \rightarrow k}-I_{\downarrow}^{k \rightarrow k+1}+\dot{\Psi}_{s}^{k}\right)
\end{aligned}
$$

where we have introduce the chemical potentials $\mu_{s}^{k} \equiv \mu_{s \uparrow}^{k}+\mu_{s \downarrow}^{k} / 2, \mu_{\downarrow}^{k} \equiv \mu_{s \downarrow}^{k} / 2+\mu_{d \downarrow}^{k}$, and the chemical affinities of the reactions, defined by $\Delta \mu_{s}^{k} \equiv \mu_{s \uparrow}^{k}-\mu_{s \downarrow}^{k} / 2=-\frac{\partial E^{k}}{\partial \Psi_{s}^{k}}, \Delta \mu_{\downarrow}^{k} \equiv$ $\mu_{s \downarrow}^{k} / 2-\mu_{d \downarrow}^{k}=-\frac{\partial E^{k}}{\partial \Psi_{s d}^{k}}$.

The entropy being an extensive variable, the total entropy variation of the system is obtained by summation over the layers 1 to $\Omega$ where the layer 1 is in contact to the left reservoir $R^{l}$ and the layer $\Omega$ is in contact to the right reservoir $R^{r}$.

The total entropy variation is:

$$
\begin{aligned}
T \frac{d S}{d t}= & {[P]^{R^{l} \rightarrow 1}-[P]^{\Omega \rightarrow R^{r}} } \\
& +\sum_{k=2}^{\Omega} \frac{1}{2}\left(\Delta \mu_{s}^{k-1}-\Delta \mu_{s}^{k}\right) \delta I_{s}^{k-1 \rightarrow k}+\sum_{k=2}^{\Omega} \frac{1}{2}\left(\mu_{s}^{k-1}-\mu_{s}^{k}\right) I_{0 s}^{k-1 \rightarrow k} \\
& +\sum_{k=2}^{\Omega} \frac{1}{2}\left(\Delta \mu_{\downarrow}^{k-1}-\Delta \mu_{\downarrow}^{k}\right) \delta I_{\downarrow}^{k-1 \rightarrow k}+ \\
& \sum_{k=2}^{\Omega} \frac{1}{2}\left(\mu_{\downarrow}^{k-1}-\mu_{\downarrow}^{k}\right) I_{0 \downarrow}^{k-1 \rightarrow k}+\sum_{k=1}^{\Omega} \Delta \mu_{s}^{k} \dot{\Psi}_{s}^{k}+\sum_{k=1}^{\Omega} \Delta \mu_{\downarrow}^{k} \dot{\Psi}_{s d}^{k}
\end{aligned}
$$

where $[P]^{R_{l} \rightarrow 1}$ and $[P]^{\Omega \rightarrow R_{r}}$ in the right hand side of the equality stand for the heat and chemical transfer from the reservoirs to the system $\Sigma$. 
The entropy variation takes the form

$$
T \frac{d S}{d t}=\sum_{i} F_{i} \dot{X}^{i}+P^{e x t}(t)
$$

where $F_{i}$ are generalized forces and $\dot{X}^{i}$ are the conjugate generalized fluxes. The variation of entropy is composed of an external entropy variation $P^{e x t}(t) / T$ and by an internal entropy variation $\mathcal{I}$.

By applying the second law of thermodynamics $\mathcal{I} \geq 0$ we are introducing the kinetic coefficients $L_{i j}$ such that $\mathcal{I}=\sum_{i} F_{i}\left(\sum_{j} L_{i j} F^{j}\right)$. By identification with the expression (B146), the kinetic equations are obtained, after performing the continuous limit,

$$
\left[\begin{array}{c}
J_{0 s} \\
J_{0 \downarrow} \\
\delta J_{s}^{d} \\
\delta J_{\downarrow}^{d} \\
\dot{\psi}_{s} \\
\dot{\psi}_{\downarrow}
\end{array}\right]=\left[\begin{array}{cccccc}
L_{s s} & L_{s \downarrow} & 0 & 0 & 0 & 0 \\
L_{\downarrow s} & L_{\downarrow \downarrow} & 0 & 0 & 0 & 0 \\
0 & 0 & L_{\delta s \delta s} & L_{\delta s \delta \downarrow} & 0 & 0 \\
0 & 0 & L_{\delta \downarrow \delta s}^{d} & L_{\delta \downarrow \delta \downarrow}^{d} & 0 & 0 \\
0 & 0 & 0 & 0 & L_{i n t}^{s} & 0 \\
0 & 0 & 0 & 0 & 0 & L_{i n t}^{\downarrow}
\end{array}\right]\left[\begin{array}{c}
\frac{-\partial \mu_{s}}{\partial z} \\
\frac{-\partial \Delta \mu_{s}}{\partial z} \\
\frac{-\partial \mu \delta}{\partial z} \\
\frac{-\partial \Delta \mu_{\downarrow}}{\partial z} \\
\Delta \mu_{s} \\
\Delta \mu_{\downarrow}
\end{array}\right]
$$

The kinetic coefficients are state functions; $L_{i j}=L_{i j}\left(S^{k}, N_{+}^{k}, N_{-}^{k}\right)$ and the symmetrized matrix is positive : $\frac{1}{2}\left\{L_{j i}+L_{i j}\right\}_{\{i j\}} \geq 0$. The coefficients $L_{i n t}$ refer to the internal relaxation processes [35, 38]. According to Onsager reciprocity relations, the kinetic coefficients are symmetric or antisymmetric $L_{i j}= \pm L_{j i}$. The coefficients are known from the two-channel model for the conductivity. The two last equations concern the internal $\left(L_{\text {int }}\right)$ "density" variables $\psi_{s}$ and $\psi_{s d}$ defined by $\Psi^{k}=\int_{\Sigma^{k}} \psi(z) d z$. Due to the Curie principle, there is no coupling between spin polarized transport processes and the electronic transitions (the scalar process is not coupled to vectorial processes).

[1] F. J. Albert, J. A. Katine, R. A. Buhrman, and D. C. Ralph, Appl. Phys. Lett. 773809 (2000).

[2] E. B. Myers, F. J. Albert, J.C. Sankey, E. Bonet, R. A. Buhrman, and D. C. Ralph, Phys. Rev. Lett. 89, 196801 (2002). 
[3] J. Grollier, V. Cros, A. Hamzic, J.M. George, H. Jaffes, A. Fert, G. Faini, J. Ben Youssef, and H. Le Gall, Appl. Phys. Lett. 78, 3663 (2001),

[4] J. Z. Sun, D. J. Monsma, D. W. Abraham, M. J. Rooks, and R. H. Koch, Appl. Phys. Lett. 81, 2202 (2002).

[5] J.-E. Wegrowe, X. Hoffer, Ph. Guittienne, A. Fabian, J.-Ph. Ansermet, and E. Olive Appl. Phys. Lett. 80, 3775 (2002).

[6] B. Oezyilmaz, A. D. Kent, D. Monsma, J. Z. Sun, M. J. Rooks, and R. H. Koch, Phys. Rev. Lett 91, 067203 (2003).

[7] A. Deac, K. J. Lee, Y. Liu, O. Redon, M. Li, P. Wang, J.-P. Nozières, and B. Dieny, Phys. Rev. B, 73, 064414 (2006).

[8] J-E. Wegrowe, D. Kelly, Y. Jaccard, Ph. Guittienne, J-Ph. Ansermet Europhysics letters 45 626 (1999).

[9] D. Kelly, J. -E. Wegrowe, Trong-kha Truong, X. Hoffer, Ph. Guittienne, and J. -Ph. Ansermet. Phys. Rev. B 68134425 (2003).

[10] J. -E. Wegrowe, M. Dubey, T. Wade, H. -J. Drouhin, and M. Konczykowski, J. Appl. Phys. 964490 (2004).

[11] M. Tsoi, A.G. M. Jansen, J. Bass, W.-C. Chiang, M. Seck, V. Tsoi, and P. Wyder, Phys. Rev. Lett. 80, 4281 (1998) and Nature 406, 6791 (2000).

[12] M. Tsoi, J. Z. Sun, M. J. Rooks, R. H. Koch, and S. S. P. Parkin, Phys. Rev. B 69, 100406(R) (2004).

[13] Y. Ji, C. L. Chien, and M. D. Stiles, Phys. Rev. Lett. 90, 106601 (2003), Phys. Rev. B, 72, $014446(2005)$.

[14] W. H. Rippard, M. R. Pufall, S. Kaka, S. E. Russek, and T. J. Silva, Phys. Rev. Lett. 92, 027201 (2004).

[15] L. Berger, J. appl. Phys. 55, 1954 (1984), P. P. Freitas and L. Berger, J. Appl. Phys. 57, 1266 (1985).

[16] J. Grollier, D. Lacour, V. Cros, A. Hamzic, A. Vaures, and A. Fert, J. Appl. Phys. 924825 (2002).

[17] N. Vernier, D. A. Allwood, D. Atkinson, M. D. Cooke, and R. P. Cowburn, Europhys. Lett. 65, $526(2004)$.

[18] M. Klaeui, C.A.F Vaz, J.A.C. Bland, W. Wernsdorfer, G. Faini, E. Cambril, LJ Heyderman, 
F. Nolting and U. Ruediger, Phys. Rev. Lett. 94, 106601 (2005).

[19] Y.-C. Chen, Y.-A. Lin, D.-C. Chen, Y.-D. Yao, S.-F. Lee, and Y. Liou, J. Appl. Phys. 97 10J703 (2005).

[20] L. Thomas, M. Hayashi, X. Jiang,R. Moriya, C. Rettner, S.S.P. Parkin.

[21] S. Urazhdin, O. Norman, W. Birge, W. P. Pratt, and J. Bass, Phys. Rev. Lett. 92, 146803 (2003); S. Urazhdin, H. Kurt, W. P. Pratt, and J. Bass, Appl. Phys. Lett. 83, 114 (2003).

[22] J.-E. Wegrowe, Phys. Rev. B 68, 214414 (2003).

[23] A. Fabian, C. Terrier, S. Serrano Guisan, X. Hoffer, M. Dubey, L. Gravier, J.-Ph. Ansermet, and J.-E. Wegrowe, Phys. Rev. Lett. 91, 257209 (2003).

[24] M. R. Pufall, W. H. Rippard, S. Kaka, S. E. Russek, and T. J. Silva, Phys. Rev. B 69, 214409 (2004)

[25] Ph. Guittienne, J.-E. Wegrowe, D. Kelly, and J.-Ph. Ansermet, IEEE Trans. Mag. Magn-37, 2126 (2001), Ph. Guittienne, L. Gravier, J.-E. Wegrowe, and J.-Ph Ansermet J. Appl. Phys. 92, 2743 (2002); and J.-E. Wegrowe, X. Hoffer, Ph. Guittienne, A. Fabian, L. Gravier, T. Wade, J-Ph. Ansermet, J. Appl. Phys 91 , 6806 (2002).

[26] S. I. Kiselev, J. C. Sankey, I. N. Krivorotov, N. C. Emley, R. J. Schoelkopf, R. A. Buhrman, and D. C. Ralph, Nature 425, 380 (2003).

[27] M. Covington, M. AlHajDarwish, Y. Ding, N. J. Gokemeijer, and M. A. Seigler, Phys. Rev. B, 69, 184406 (2004).

[28] I.N. Krivorotov, N.C. Emley, J.C. Sankey, S.I. Kiselev, DC. Ralph, R. A. Buhrman, Science 30728 (2005).

[29] L. Berger Phys. Rev. B 54, 9353 (1996), L. Berger J. Appl. Phys. 81, 4880 (1997).

[30] J. C. Slonczewski, J. Magn. Magn. Mat. 159 L1 (1996).

[31] I. Prigogine, P. Mazur, Physica 19, 241 (1953).

[32] Dilip Kondepudi, Ilya Prigogine, Modern thermodynamics, John Wiley and Sons Ltd, 1998.

[33] E. A. Guggenheim, Thermodynamics, North Holland, 1969.

[34] E.C.G. Stueckelberg and P.B. Scheurer, thermocinétique phénoménologique galiléenne" Birkauser Verlag, Basel and Stuttgart, 1974.

[35] S. R. De Groot and P. Mazur, non equilibrium thermodynamics Amsterdam : North-Holland, 1962.

[36] J. Kuiken, Thermodynamics of irreversible processes, J. Wiley, 1994. 
[37] J. E. Parrott, IEEE Trans. Electron Devices 43, 809 (1996).

[38] P. Mazur, Physica A 261, 451 (1998).

[39] Ch. Gruber, Eur. J. Phys. 20, 259 (1999).

[40] J. M. G. Vilar and J. M. Rubí, Proc. Nat. Acad. Sci. USA, 98, 11081 (2001).

[41] A. Pérez-Madrid, T. Alarcón, J.M.G Vilar, J.M. Rubí, Physica, 270, 403 (1999).

[42] J.-E. Wegrowe, Phys. Rev. B 62, 1067 (2000).

[43] J.-E. Wegrowe, Q. Anh Nguyen, M. Al-Barki, J.-F. Dayen, T. L. Wade, and H.-J. Drouhin, Phys. Rev. B 73 134422, (2006).

[44] J.-E. Wegrowe, H.-J. Drouhin, Proc. SPIE Quantum Sensing and Nanophotonic Devices II Vol. 5732, 498 (2005), J.-E. Wegrowe, and H.-J. Drouhin, arXiv:cond-mat/0408410

[45] the coefficient $\nu_{i j}$ is defined by the stochiometric coefficients. If the chemical reaction $i$ writes $\nu_{i A}^{\prime} A \rightarrow \nu_{i A}^{\prime \prime} A$, with stochiometric coefficients $\nu_{i A}^{\prime}$ and $\nu_{i A}^{\prime \prime}$, the coefficent $\nu_{i A}$ apprearing in the continuity equations is $\nu_{i A}=\nu_{i A}^{\prime \prime}-\nu_{i A}^{\prime}$.

[46] In what follows, there is no kinetic energy, i.e. no inertial effects. This is the reason why the equations are reduced to the simplest expression.

[47] Théophile De Donder, l'Affinité, Gauthier-Villars, Paris 1927.

[48] L. Onsager, the collected work of Lars Onsager. With Commentary, Edited by P. C. Hemmer, H. Holden, and S. Kjelstrup Ratkje, World Scientific Series in 20 th century Physics, col. 17, World Scientific Publishing, New Jersey, 1996.

[49] J. Shi, S. S. P. Parkin, L. Xing, M. B. Salamon, J. Appl. Phys. 73, 5524 (1993).

[50] L. Gravier, S. Serrano-Guisan, F. Reuse, and J. -Ph. Ansermet, Phys. Rev. B 73, 024419 (2005).

[51] M. B. Stearns, Phys. Rev. B, 8, 4383 (1973).

[52] N. F. Mott and H. Jones, Theory of the properties of metal and alloys, Oxford University Press, 1953.

[53] A. Fert, I.A. Cambell, J. Phys. F: Met. Phys. 6, 849 (1976).

[54] R. Potter, Phys. Rev. B 10, 4626 (1974).

[55] T. R. McGuire and R. I. Potter, IEEE Trans. vol Mag-11, 1018 (1975).

[56] H.-J. Drouhin Spin-dependent scattering in transition metals, J. Appl. Phys. 89, 6805 (2001).

[57] F. J. Blatt, P. A. Schroeder, C. L. Foiles, and D. Greig, Termoelectric power of metals, Plenum Press, 1976, Chap. 5. 
[58] M. N. Baibich, J. M. Broto, A. Fert, F. Ngyen Van Dau, F. Petroff, P. Etienne, G. Creuzet, A. Friederich, and J. Chazelas, Phys. Rev. Lett. 61, 2472 (1988) and G. Binasch, P. Grunberg, F. Saurenbach, and W. Zinn, Phys. Rev. B 39, 4828 (1989).

[59] D. D. Awschalom and J. M. Kikkawa, Phys. Today 53, 33 (1999).

[60] M. Viret, D. Vignoles, D. Cole, J. M. D. Coey, W. Allen, D.S. Daniel, and J.F. Gregg, Phys. Rev. B 53, 8464 (1996).

[61] P.M. Levy and S. Zhang Phys. Rev. Lett. 79, 5110 (1997).

[62] U. Ruediger, J. Yu, L. Thomas, S.S.P. Parkin, and A.D. Kent, Phys. Rev. B 59, 11914 (1999).

[63] J. -E. Wegrowe, A. Comment, Y. Jaccard, J.- Ph. Ansermet, N. M. Dempsey, and J. -Ph. Nozières, Phys. Rev B, 6112216 (2000).

[64] M.A.M. Gijs, G.E.W Bauer, Adv. Phys. 46, 285 (1997).

[65] R. K. Nesbet, IBM J. Develop., 42, 53 (1998).

[66] P. M. Levy, I. Mertig, Spin dependent transport in magnetic nanostructures, S. Maekawa, T. Shino (Eds), Taylor and Francis, London, (2002).

[67] I. Zutić, J. Fabian, S. Das Sarma, Rev. Mod. Phys. 76323 (2004).

[68] G. Schmidt, J. Phys. D: Appl. Phys. 38, R107 (2005).

[69] Ch. Marrows, Adv. Phys. 54, 585 (2005).

[70] M. Johnson and R.H. Silsbee Phys. Rev. B 35, 4959 (1987); M. Johnson and R. H. Silsbee, Phys. Rev. B 37, 5312 (1988).

[71] P. C. van Son, H. van Kempen, and P. Wyder, Phys. Rev Lett. 58, 2271 (1987).

[72] T. Valet and A. Fert, Phys. Rev. B, 48, 7099 (1993).

[73] P. M. Levy, H. E. Camblong, S. Zhang, J. Appl. Phys. 75, 7076 (1994).

[74] A. Fert, J.-L. Duvail, Th. Valet, Phys. Rev. B 52, 6513 (1995).

[75] C. Heide, P.E. Zil'berman, and R. J. Elliott, Phys. Rev. B, 63, 064424 (2001), C. Heide, Phys. Rev. B 65, 054401 (2001).

[76] G. Schmidt, D. Ferrand, L. W. Molenkamp, A. T. Filip, and B. J. van Wees, Phys. Rev. B 62, R4790 (2000).

[77] F. J. Jedema, B. J. van Wees, B. H. Hoving, A. T. filip, and T. M. Klapwijk, Phys. Rev. B 60, 16549 (1999).

[78] Motofumi Suzuki and Yasunori Taga, Phys. Rev. B, 52, 361 (1995). 
[79] E. Y. Tsymbal, D. G. Pettifor, J. Shi, M. B. Salamon, Phys. Rev. B 59, 8371 (1999).

[80] R. J. Baxter, D. G. Pettifor, E. Y. Tsymbal, D. Bozec, J. A. D. Matthew, and S. D. Thomson, J. Phys. : Condens. Matter 15, L695 (2003).

[81] J. -E. Wegrowe, H. -J. Drouhin, Proc. SPIE Quantum Sensing and Nanophotonic Devices II Vol. 5732, 498 (2005), and J. -E. Wegrowe and H. -J. Drouhin, cond-mat/0408410 (2005).

[82] A. Fert, Shang-Fan Lee, J. Magn. Magn. Mat. 165, 115 (1997).

[83] In all these calculations, the channel parameters $\sigma_{\alpha \gamma}, \beta$, and $L$ are considered as constant in space. It can be shown that taking into account the gradient only introduces vanishingly small corrections.

[84] The Riemann integral is evaluated in the intervals where the derivative is regular. Note that a global calculation over $[A, B]$, within the framework of the distribution theory, yields zero because $\Delta \mu(A)=\Delta \mu(B)=0$. This simply means that the integral of the regular part is opposite to the integral of the Dirac masses located at the interface. Let us write that the out-of-equilibrium resistance is straightforwardly connected to the chemical potential drop between the $\mathrm{A}$ and $\mathrm{B}$ points, diminished by the standard potential drop which would result from the application of Ohm's law. We obtain : $J_{t} e R^{n e}=\left[\mu_{I}(A)-\mu_{I I}(B)\right]-e\left[\Phi_{I}(A)-\Phi_{I}\left(0^{-}\right)\right]-e\left[\Phi_{I I}\left(0^{+}\right)-\Phi_{I I}(B)\right]=\left[\mu_{I}(A)-e \Phi_{I}(A)\right]-$ $\left[\mu(0)-e \Phi_{I}\left(0^{-}\right)\right]+\left[\mu(0)-e \Phi_{I I}\left(0^{+}\right)\right]-\left[\mu_{I I}(B)-e \Phi_{I I}(B)\right]=e\left[\Phi_{I}\left(0^{-}\right)-\Phi_{I I}\left(0^{+}\right)\right]$. It can be seen that the second expression for $J_{t} e R^{n e}$ above is nothing but the opposite of Eq.(6), and is equal to the discontinuity of $\Phi$ at the interface, which provides a simple physical interpretation as illustrated in Fig. 3 .

[85] F. J. Jedema, A. T. Filip, B. J. van Wees, Nature 410, 345 (2001)

[86] J. -M. George, G. Faini and A. Fert, Phys. Rev. B 67, 012410 (2003).

[87] E. I. Rashba, Phys. Rev. B 62, R16267 (2000).

[88] L. Néel, Ann. Geophys. 5, 99 (1949).

[89] W. F. Brown Jr., Phys. Rev. 130, 677 (1963).

[90] W. F. Brown Jr., Micromagnetics, Interscience publishers, 1963.

[91] W. T. Coffey, Yu. P. Kalmykov and J. T. Waldron, The Langevin equation, World Scientific Series in contemporary Chemical Physics Vol. 11, 1996.

[92] J. L. Garcia-Palacios and F. J. Lazaro, Phys. Rev. B 58, 14937 (1998).

[93] W. T. Coffey, D.S.F. Crothers, J.L. Dormann, Yu. P. Kalmikov, E.C. Kennedy, and W. 
Wernsdorfer, Phys. Rev. Lett. 80, 5655 (1998).

[94] W. Wernsdorfer, E. Bonnet Orozco, K. H. Hasselbach, A. Benoit, B. Barbara, N. Demoncy, A. Loiseau, H. Pascard, and D. Mailly, Phys. Rev. Lett. 78, 1791 (1997).

[95] J.-E. Wegrowe, O. Fruchart, J.-P. Nozières, F.Rousseaux, D.Decanini, J.-Ph. Ansermet, and D. Givord, J. Appl. Phys. 861028 (1999).

[96] Z. Jin, N. Bertram, IEEE Trans. Magn 38, 2265 (2002).

[97] N. Smith, V. Synogatch,D. Mauri,J.A. Katine, and M.-C. Cyrille, J. Appl. Phys. 917454 (2002).

[98] H. W. Schumacher, C. Chappert, R.C. Sousa, P. P. Freitas, J. Miltat, J. Ferré, J. Appl. Phys. 93, 7290

[99] H. W. Schumacher, C. Chappert, R. C. Sousa, P. P. Freitas, J. Miltat, Phys. Rev. Lett 90, 017204 (2003); and H. W. Schumacher,C. Chappert, R. C. Sousa, P. P. Freitas Appl. Phys. Lett. 83, 2205 (2003).

[100] S. Kaka, J. P. Nibarger and S. E. Russek, N.A. Stutzke, S. L. Burkett, J. Appl. Phys. 93, $7539(2003)$

[101] M. Sparks, Ferromagnetic-relaxation theory, McGraw-Hill, Advenced physics monograph series, New-York 1964.

[102] A. Aharoni, Introduction to the Theory of Ferromagnetism (Clarendon Press, Oxford) 1996.

[103] J. Kurkijärvi, Phys. Rev. B 6, 832 (1972).

[104] Y. Acremann, J.-P. Strachan, V. Chembrolu, S. D. Andrews, T. Tyliszczak, J. A. Katine, M. J. Carey, B. M. Clemens, H. C. Siegmann, and J. Stoehr, Phys. Rev. Lett. 96, 137206 (2006).

[105] V. Kamberskỳ, Can. J. Phys. 48, 2906 (1970).

[106] D. Forster, Hydrodynamics, Fluctuations, Broken Symmetry, and Correlation Functions, Frontiers in Physics, 1975 W. A: Benjamin Inc.

[107] E. Fick and S. Sauermann, the quantum statistics of Dynamic Processes, Springer Series in Solid-States Siences 86, Ed. M. Cordona, P. Fulde, K. von Klitzing, H.-J. Queisser, Berlin Heidelberg 1990.

[108] P. Hänggi, P. Talkner, and M. Borkovec, Reaction rate theory: fifty years after Kramers, Rev. Mod. Phys. 62, 251 (1990).

[109] R. Balian, Am. J. Phys. 67, 1078 (1999). 
[110] Z. Li and S. Zhang, Phys. Rev. B, 69, 134416 (2004).

[111] D.M. Apalkov and P.B. Visscher, J. Magn. Magn. Mat. 286370 (2005).

[112] C. Serpico, G. Bertotti, I. D. Mayergoyz, M. D’Aquino, R. Bonin, J. Appl. Phys. 99, 08G505 (2006).

[113] Y. Yuzhelevski et al. 71, 16811 Rev. Sc. Instr. (2000).

[114] J. G. Zhu and X. Zhu, IEEE Trans. Magn. 40, 182 (2004).

[115] K. J. Lee, A. Deac, O. Redon, J. P. Nozieres, and B. Dieny,Nat. Matter 3, 877 (2004).

[116] H. B. Callen, J. Phys. Chem. Sol. 4, 256 (1958).

[117] Ya. B. Bazaliy, B.A. Jones, and S.-C. Zhang, Phys. Rev. B, 57, R3213 (1998).

[118] X. Waintal , E. B. Myers, P. W. Brouwer, and D. C. Ralph, Phys. Rev. B 62, 12317 (2000).

[119] S. Zhang, P. M. Levy, and A. Fert, Phys. Rev. Lett. 88, 236601 (2002); A. Shpiro, P. M. Levy, and S. Zhang, Phys. Rev. B 67, 104430 (2003).

[120] M. D. Stiles, J. X. Xiao, and A. Zangwill, Phys. Rev. B 69, 054408 (2004); M. D. Stiles and A. Zangwill, Phys. Rev. B 66, 014407 (2002); M. D. Stiles and A. Zangwill, J. Appl. Phys. 91, 6812 (2002),

[121] A. Brataas, Yu. Nazarov, and G.E.W Bauer, Phys. Rev. Lett. 84, 2481 (2000); D. Harnando, Y. V. Nazarov, A. Brataas, and G.E.W Bauer, Phys. Rev. B 62, 5700 (2000); Y. Tserkovnyak, A. Brataas, and G. E. W. Bauer, Phys. Rev. Lett. 88, 117601 (2002); G. E.W. Bauer, Y. Tserkovnyak, D. Huertas-Hernando, and A. Brataas, Phys. Rev. B 67, 094421 (2003).

[122] M. L. Polianski and P. W. Brouwer, Phys. Rev. Lett. 92, 026602 (2004).

[123] J. Casas-Vazquez, D. Jou, Temperature in non-equilibrium states: a review of open problems and current proposals Rep. Prog. Phys. 661937 (2003).

[124] E. M. Purcell and R. V. Pound, Phys. Rev. 81, 279 (1951).

[125] N. F. Ramsey, Phys. Rev. 103, 20 (1956).

[126] A. Abragam and W. G. Proctor, Pys. Rev. 109, 1441 (1958).

[127] J. Kunes and V. Kamberskỳ, Phys. Rev. B, 65, 212411 (2002).

[128] H. Shul, J. Appl. Phys. 89, 7448 (2001).

[129] According to Eq. (18), the value of $\Delta \mu$ is of the order of the giant magnetoresistance multiplied by the current, $\Delta$ R.I, i.e. of the order of tens of $\mu \mathrm{eV}$ (up to $\mathrm{meV}$ in CIMS experiments).

[130] S. Urazhdin, O. Norman, W. P. Pratt Jr. and J. Bass J. Appl. Phys. 95, 7429 (2004).

[131] Y. Jiang, S. Abe, T. Ochiai, T. Nozaki, A. Hirohata, N. Tezuka, and K. Inomata, Phys. Rev. 
Lett. 92, 167204 (2004). 